\title{
Title: Structures of C1-IgG1 provide insights into how danger pattern recognition activates complement
}

Authors: Deniz Ugurlar'1, Stuart C. Howes², Bart-Jan de Kreuk ${ }^{3}$, Roman I. Koning ${ }^{2,4}$, Rob N. de Jong $^{3}$, Frank J. Beurskens ${ }^{3}$, Janine Schuurman ${ }^{3}$, Abraham J. Koster ${ }^{2,4}$, Thomas H. Sharp ${ }^{2 *}$, Paul W.H.I. Parren ${ }^{3,5 *}$, Piet Gros ${ }^{1 *}$

\begin{abstract}
Affiliations:
${ }^{1}$ Crystal and Structural Chemistry, Bijvoet Center for Biomolecular Research, Department of Chemistry, Faculty of Science, Utrecht University, Padualaan 8, 3584 CH Utrecht, The Netherlands.
\end{abstract}

${ }^{2}$ Section Electron Microscopy, Department of Molecular Cell Biology, Leiden University Medical Center, Einthovenweg 20, 2300 RC Leiden, The Netherlands.

${ }^{3}$ Genmab, Yalelaan 60, 3584 CM Utrecht, The Netherlands.

${ }^{4} \mathrm{NeCEN}$, Gorlaeus Laboratories, Leiden University, 2333 CC Leiden, The Netherlands

${ }^{5}$ Department of Immunohematology and Blood Transfusion, Leiden University Medical Center, Albinusdreef 2, 2333 ZA Leiden, the Netherlands

*Correspondence to: Thomas H. Sharp (t.sharp@,lumc.nl), Paul W.H.I. Parren

(P.W.H.I.Parren@lumc.nl), Piet Gros (p.gros@uu.nl) 
Abstract: Danger patterns on microbes or damaged host cells bind and activate C1, inducing innate immune responses and clearance through the complement cascade. How these patterns trigger complement initiation remains elusive. Here, we present cryo-EM analyses of $\mathrm{C} 1$ bound to monoclonal antibodies in which we observed heterogeneous structures of single and clustered C1-IgG1 hexamer complexes. Distinct C1q binding sites are observed on the two Fc-CH2 domains of each IgG molecule. These are consistent with known interactions and also reveal additional interactions, which are supported by functional IgG1-mutant analysis. Upon antibody binding, the $\mathrm{Clq}$ arms condense, inducing rearrangements of the $\mathrm{C} 1 \mathrm{r}_{2} \mathrm{~s}_{2}$ proteases and tilting C1q's cone-shaped stalk. The data suggest that C1r may activate C1s within single, strained C1 complexes or between neighboring $\mathrm{C} 1$ complexes on surfaces.

One Sentence Summary: Cryo-EM structures of C1 bound to antibodies suggest mechanisms for how danger patterns on cell membranes trigger an immune response.

Main Text: The complement system is part of our innate immune system. The classical complement pathway is triggered by activation of the $\mathrm{C} 1$ initiation complex upon binding to cell surfaces. $\mathrm{C} 1$, or $\mathrm{Clqr}_{2} \mathrm{~s}_{2}$, consists of four proteases, $\mathrm{C} 1 \mathrm{r}$ and $\mathrm{C} 1 \mathrm{~s}$, that associate with $\mathrm{C} 1 \mathrm{q}$, which contains antibody-binding sites. The homologous serine proteases $\mathrm{C} 1 \mathrm{r}$ and $\mathrm{C} 1 \mathrm{~s}$ each consist of six domains (Fig. S1A). C1q comprises 18 polypeptide chains; three chains of C1q A, B and C trimerize to form six collagen-like triple helices connected to six globular (trimeric) ligandrecognition (gC1q) modules (Fig. S1B) (1). Binding of $\mathrm{C} 1$ through its gC1q modules to mediators of inflammation, such as IgG or IgM antibodies (Fig. S1C,D), on cell surfaces activates the associated proteases and initiates the proteolytic cascade of complement (2-4). 
Previously, we demonstrated that $\operatorname{IgG}$ molecules, bound to their cognate antigens on liposomes or cell membranes, oligomerize through interactions between their Fc regions and form hexameric, high avidity, C1-binding structure reminiscent of multimeric IgM antibodies (Fig. S1D) (5). Mutagenesis studies (6-8) showed that amino-acid residues in IgG1 important for direct $\mathrm{C} 1$ binding are situated in the $\mathrm{CH} 2$ domains near the $\mathrm{Fab}-\mathrm{Fc}$ hinge at the periphery of these Fc-platforms (Fig. S1C). In C1q, globular head residues of predominantly C1q B mediate $\operatorname{IgG}$ binding $(4,9,10)$. However, the molecular sequence of events leading to $\mathrm{C} 1$ activation by IgG hexamers remains poorly understood (11). Here, we used IgG monoclonal antibodies (mAb) oligomerized through antigen-binding on liposomes or preformed antibody-complexes in solution and applied tomography and single-particle cryo-electron microscopy (EM) to resolve the mechanisms of $\mathrm{C} 1$ binding and activation.

Liposomes carrying di-nitrophenyl (DNP) haptens were incubated with an anti-DNP chimeric IgG1 mAb and $\mathrm{C} 1$ to allow extensive formation of surface-bound C1-IgG1 complexes (Fig. 1A). Tomograms showed marked structural variations in $\mathrm{C} 1$ binding to antibodies on these liposomes (Fig. 1A, Fig S2A,B). Alignment and classification of single membrane-bound C1-IgG1 complexes (Fig. 1B) yielded a reconstruction at $\sim 25-\AA$ A resolution (Fig. S2C,D). Focused alignment and classification on the $\mathrm{Fc}-\mathrm{C} 1$ complex (excluding the membrane and $\mathrm{Fab}$ domains; Fig. S2B,E) revealed six densities corresponding to gC1q domains binding an Fc-platform formed by six IgG1 molecules, a rhomboidal platform accounting for bound $\mathrm{C}_{1} \mathrm{r}_{2} \mathrm{~S}_{2}$ proteases and a protruding Clq-collagen stalk on top (Fig. 1C), consistent with a previous reconstruction obtained with a goat polyclonal anti-DNP antibody at $\sim 65-\AA ̊$ resolution (5). Analysis of subvolumes of $\mathrm{C} 1-\mathrm{IgG} 1$ complexes revealed persistent density for neighboring $\mathrm{C} 1$ complexes (Fig. 1D, Fig. S2E), as previously observed using normal human serum (12). Distances between 
nearest neighbors varied from approx. 11-40 nm center-to-center, with a peak at $23 \mathrm{~nm}$ (Fig. $\mathrm{S} 2 \mathrm{~F}$ ), reflecting a variation of arrangements of neighboring complexes. The $\mathrm{C} 1$ complexes are not evenly distributed across the surfaces of the liposomes, suggesting that there is preference for the complexes to associate, rather than occupy all available liposome surface (Fig. 1A, S2A).

Soluble C1-IgG1 6 complexes of 1.7 MDa were obtained (Fig. S3A,B) by incubating C1, with catalytically inactive proteases $\mathrm{C} 1 \mathrm{r}$ (S654A) and C1s (S632A), with a human IgG1 mAb containing three mutations that drive the formation of $\operatorname{IgG}$ hexamers in solution (i.e. IgG1E345R, E430G and S440Y) $(5,13,14)$. Classification and averaging of single-particle densities yielded separate classes with four, five or six $\mathrm{gClq}$ domains in contact with the $\mathrm{Fc}$ platforms (Fig. 2A,B, Fig. S4C). One class containing $\sim 79,000$ particles with six gC1q domains bound to the Fc platform yielded a map at 10-Å resolution (Fig. S4D), resulting in an overall structure 32 $\mathrm{nm}$ high and $25 \mathrm{~nm}$ wide that is consistent with densities observed in tomography (Fig. S5). The reconstruction reveals densities for all $\mathrm{Clq}$ collagen-like triple helices and $\mathrm{gClq}$ modules, $\mathrm{Clr}$ and $\mathrm{C} 1 \mathrm{~s}$ proteases and $\mathrm{IgG1}-\mathrm{Fc}$ regions (Fig. 2C,D).

Imposing six-fold symmetry on the IgG1 platform bound to gC1q yielded a density map at 7.3- $\AA$ resolution (Fig. 3A, Fig. S4D). Crystal structures of Fc $\mathrm{CH} 2$ and $\mathrm{CH} 3$ domains (pdb-code 1HZH) (15) and gC1q (1PK6) (16) were modeled in this density map (Fig. 3A). In the resulting model, each $\mathrm{gClq}$ domain contacts peripheral areas on both $\mathrm{CH} 2$ and $\mathrm{CH} 2$ ' domains of an IgG-Fc dimeric segment, burying $\sim 540 \AA^{2}$ surface area (Fig. S6A). The Fc segments adopt an open conformation, characterized by a long distance of $31 \AA$ \&etween Pro329 and Pro329’ of the CH2 domains (Fig. S6B). This contrasts with observations of closed conformations in many crystal structures of Fc domains with Pro329-Pro329’ distances of $\sim 12-19 \AA$, but resembles that of fulllength IgG1-b12 (1HZH) (15) and deglycosylated Fc fragments of human IgG4 (4D2N) (17), 
both of which exhibit a six-fold (crystal) packing of their Fc portions, that have Pro329-Pro329' distances of 24 and $29 \AA$, respectively. Densities are present for N-linked glycans at Asn297 and Asn297' (Fig. 3A). However, no direct contact is observed between the glycans and gC1q, supporting the idea that glycosylation affects $\mathrm{C} 1$ binding through $\operatorname{IgG}$ hexamerization (13). Fitting of hetero-trimeric $\mathrm{gClq}$ to the density yielded similar correlation coefficients for three possible A-B-C domain orientations, with a marginally higher score for chains $\mathrm{B}$ and $\mathrm{C}$ facing the antibodies, consistent with mutation data that has identified chains $\mathrm{B}$ and $\mathrm{C}$ harboring the antibody-binding sites (9).

The $\mathrm{Fc}-\mathrm{gC} 1 \mathrm{q}$ structure identified distinct $\mathrm{C} 1 \mathrm{q}-$ binding sites on the two $\mathrm{Fc}-\mathrm{CH} 2$ domains of an IgG1. The observed binding sites are corroborated by extensive mutagenesis, which shows that both previously established amino acid contacts and contacts newly identified in our structure modulate complement activation (Fig. 3B,C; Fig. S6C) $(6-8,18)$. Mutations were introduced in the CD20 mAb IgG1-7D8 and the impact on complement-dependent cytotoxicity (CDC) of CD20-expressing Raji cells was assessed (Fig. 3C, Table S1). The first binding site is formed by loop FG (residues 325-331) of Fc $\mathrm{CH} 2$, known to be involved in binding both $\mathrm{C} 1 \mathrm{q}$ and $\mathrm{Fc}-$ receptors (18-20). Critical residues Pro329-Ala330-Pro331 $(3,6,18)$ form the tip of the FG loop with Pro329 making contact with hydrophobic C1q-B residue Phe178 (Fig. 3B). IgG1 CH2 residue Lys322 (21) provides additional charged interactions with C1q-C residue Asp195 (Fig. 3B). Mutation of Ala327 into a positively charged lysine decreased CDC, whereas the mutation Ala327Gly enhanced CDC (Fig. 3C). Consistent with previous observations, variant Lys326Ala/Glu333Ala stimulated CDC (6). The secondary binding site consists of loop BC (res. 266-272) and loop DE (res. 294-300) of CH2', which form a negatively charged patch that interacts with C1q-B residues Arg114 and Arg129 (Fig. 3B) (4, 22). Introducing a positive 
charge at residues Glu269, Glu294 or Tyr300 abolished CDC (Fig. 3C). In contrast, the mutation Tyr300Asp enhanced CDC (Fig. 3C). Mutations Asn297Gln and Ser298Lys decreased CDC, likely due to absence of glycosylation. Furthermore, Fab-Fc hinge region residues Glu233, Leu234, Leu235, Gly236 and Gly237, contributed to C1q binding and CDC (Fig. S6C). Alanine substitutions of these residues decreased CDC, whereas Gly236Asp enhanced CDC, suggesting a possible charge interaction with C1q-B Arg150. The Fab regions themselves are positioned flexibly below the Fc platform, as is apparent in the tomography reconstructions (Fig. 1B,C, Fig. S5), and appear not to contribute directly to Clq binding and CDC.

We fitted structural models of $\mathrm{C} 1 \mathrm{q}, \mathrm{C} 1 \mathrm{r}$ and $\mathrm{C} 1 \mathrm{~s}$ into the density reconstruction of $\mathrm{C} 1-\mathrm{IgG} 1_{6}$ (Fig. 4, Fig. S7). On top of the $\mathrm{C} 1$ structure, the six C1q-A, B and C collagen-like triple helices form a stalk that adopts a continuous, hollow cone-shaped structure, which is tilted by $15^{\circ}$ from the vertical axis. Six triple helices emerge from the stalk, extend downwards (with an irregular small right-handed supercoil) and connect to the $\mathrm{gClq}$ modules that bind the $\mathrm{IgG} 1-\mathrm{Fc}$ hexamer platform. In particular, the collagen-like helices 3 and 6 display a marked bending (Fig. 4A). Density positioned in between the collagen-like helices is consistent with previously proposed binding of $\mathrm{N}$-terminal domains of $\mathrm{C}_{1} \mathrm{r}_{2} \mathrm{C}_{1} \mathrm{~s}_{2}$ between the $\mathrm{C} 1 \mathrm{q}$ arms, with arms 2, 3, 5 and 6 contacting C1r and arms 1 and 4 contacting C1s molecules (23-25) (Fig. 4B). Using crystal structures of C1r and C1s (25-28), and their homologs MASP1 and MASP2 (29, 30), domains CUB1-EGF-CUB2-CCP1 of both C1r and C1s (Fig. S1A) were modeled into the densities (Fig. S7). No density is observed for the CCP2-SP domains of either C1r or C1s in the 10- $\AA$ resolution single-particle reconstruction, indicating flexible arrangements for these parts. However, density obtained for CCP1 domains allows completion of the model by superposition of CCP1-CCP2-SP crystal structures onto CCP1 of C1r and C1s (Fig. 4C, Fig. S1A). This results in a model in 
which $\mathrm{C} 1 \mathrm{r} \mathrm{CCP} 1$ orients $\mathrm{CCP} 2-\mathrm{SP}$ to curve around the $\mathrm{C} 1 \mathrm{q}$ collagen-like helix towards $\mathrm{C} 1 \mathrm{~s}$ and in which $\mathrm{C} 1 \mathrm{~s} \mathrm{CCP} 1-\mathrm{CCP} 2-\mathrm{SP}$ sticks outwards, consistent with their proteolytic functions in the complement cascade (Fig. 4C Fig. S7D).

The observed arrangement of the $\mathrm{C} 1 \mathrm{r}$ and $\mathrm{C} 1 \mathrm{~s}$ hetero-tetramer differs from predictions based on a tetrameric $\mathrm{C} 1 \mathrm{~s}$ arrangement $(25,31)$ The CUB2 domains of $\mathrm{C} 1 \mathrm{r}$ and $\mathrm{C} 1 \mathrm{~s}$ are rotated and the C1r-C1s dimers are shifted along each other, shortening the contact sites of C1q-collagen helices 2 and 5 from 14 (31) to $11 \mathrm{~nm}$ in C1-IgG1 6 (Fig. S7A-C). The arrangement of the C1q arms, induced upon binding the $\mathrm{Fc}$ hexamer, is also indicative of a compaction. The $\mathrm{gC} 1 \mathrm{q}$ domains in unbound $\mathrm{C} 1$ are spread apart up to $30-35 \mathrm{~nm}(31)$. Bending of the collagen-like helices of arms 3 and 6, which embrace $\mathrm{C}_{1} \mathrm{r}_{2} \mathrm{~s}_{2}$ in the longest dimension, and incomplete binding of the $\mathrm{gClq}$ heads (on arms 5 and 6) to Fc platforms support the notion of a surface-induced conformational change.

The affinity of $\mathrm{gClq}$ modules for single IgG antibody molecules is very low. For IgG antibody molecules to form a recognition pattern therefore requires their clustering or aggregation, allowing the formation of a multivalent complex with $\mathrm{C} 1$. IgM molecules are already multivalent, but require their occluded $\mathrm{C} 1$ binding sites to be revealed upon interacting with surface antigen. Here we show that the multivalent binding of $\mathrm{C} 1$ to IgG hexamers results in compaction of C1q arms, which rearranges the N-terminal (CUB1-EGF-CUB2) platform of the $\mathrm{C}_{2} \mathrm{r}_{2} \mathrm{~S}_{2}$ proteases, that may allow the catalytic SP domain of the C1r CCP1-CCP2-SP arm to reach the scissile loop in C1s CCP1-CCP2-SP. Alternatively, the extended conformations of the CCP1-CCP2-SP domains may allow inter-complex proteolysis induced by neighboring complexes. This is consistent with the $\mathrm{C} 1$-antibody complexes that form on crowded surfaces, as observed in tomograms of $\operatorname{IgG} \mathrm{mAb}$ hexamers bound to liposomes. Intercomplex activation has 
been proposed for MBL-MASP $2_{2}$ complexes of the lectin-binding complement pathway, in which MASP1 proteases present in separate MBL-MASP $1_{2}$ complexes mediate activation (31, 32). Direct binding of $\mathrm{C} 1$ to ubiquitous and fluid ligands in a membrane, such as

phosphatidylserines on apoptotic cells, would likely not induce compaction of the C1q arms and activation may depend on intercomplex proteolysis of surface-bound $\mathrm{C} 1$ complexes. Our data suggest that danger pattern recognition by $\mathrm{C} 1$ may lead to proteolysis and activation within an isolated complex through a conformational change as suggested by an observed bending of $\mathrm{Clq}$ arms and the arrangement of proteases. Close interactions observed between separate C1-IgG complexes however suggest that proteolysis may also result from intercomplex activation.

\section{References}

1. G. J. Arlaud et al., Structural biology of C1: dissection of a complex molecular machinery. Immunol. Rev. 180, 136-145 (2001).

2. N. C. Hughes-Jones, B. Gardner, Reaction between the isolated globular sub-units of the complement component Clq and IgG-complexes. Mol. Immunol. 16, 697-701 (1979).

3. D. R. Burton et al., The Clq receptor site on immunoglobulin G. Nature. 288, 338-344 (1980).

4. L. T. Roumenina et al., Interaction of $\mathrm{C} 1 \mathrm{q}$ with $\mathrm{IgG} 1, \mathrm{C}-$ reactive protein and pentraxin 3: mutational studies using recombinant globular head modules of human $\mathrm{Clq}$ A, B, and C chains. Biochemistry. 45, 4093-4104 (2006).

5. C. A. Diebolder et al., Complement is activated by IgG hexamers assembled at the cell surface. Science. 343, 1260-3 (2014). 
6. E. E. Idusogie et al., Engineered antibodies with increased activity to recruit complement. J. Immunol. 166, 2571-2575 (2001).

7. A. R. Duncan, G. Winter, The binding site for C1q on IgG. Nature. 332, 738-740 (1988).

8. G. L. Moore, H. Chen, S. Karki, G. A. Lazar, Engineered Fc variant antibodies with enhanced ability to recruit complement and mediate effector functions. MAbs. 2, 181-189 (2010).

9. M. S. Kojouharova et al., Mutational analyses of the recombinant globular regions of human $\mathrm{Clq} \mathrm{A}, \mathrm{B}$, and $\mathrm{C}$ chains suggest an essential role for arginine and histidine residues in the C1q-IgG interaction. J. Immunol. 172, 4351-4358 (2004).

10. U. Kishore et al., Structural and functional anatomy of the globular domain of complement protein C1q. Immunol. Lett. 95, 113-28 (2004).

11. C. Gaboriaud, W. L. Ling, N. M. Thielens, I. Bally, V. Rossi, Deciphering the fine details of $\mathrm{C} 1$ assembly and activation mechanisms: "Mission impossible"? Front. Immunol. 5, 565 (2014).

12. T. H. Sharp, F. G. A. Faas, A. J. Koster, P. Gros, Imaging complement by phase-plate cryo-electron tomography from initiation to pore formation. J. Struct. Biol. 197, 155-162 (2016).

13. G. Wang et al., Molecular basis of assembly and activation of complement component $\mathrm{C} 1$ in complex with immunoglobulin G1 and antigen. Mol. Cell. 63, 135-145 (2016).

14. R. N. de Jong et al., A novel platform for the potentiation of therapeutic antibodies based on antigen-dependent formation of $\operatorname{IgG}$ hexamers at the cell surface. PLoS Biol. 14 (2016), doi:10.1371/journal.pbio.1002344.

15. E. O. Saphire et al., Crystal structure of a neutralizing human IGG against HIV-1: a 
template for vaccine design. Science. 293, 1155-1159 (2001).

16. C. Gaboriaud et al., The crystal structure of the globular head of complement protein C1q provides a basis for its versatile recognition properties. J. Biol. Chem. 278, 46974-82 (2003).

17. A. M. Davies, R. Jefferis, B. J. Sutton, Crystal structure of deglycosylated human IgG4Fc. Mol. Immunol. 62, 46-53 (2014).

18. E. E. Idusogie et al., Mapping of the $\mathrm{C} 1 \mathrm{q}$ binding site on rituxan, a chimeric antibody with a human IgG1 Fc. J. Immunol. 164, 4178-84 (2000).

19. M. H. Tao, R. I. Smith, S. L. Morrison, Structural features of human immunoglobulin G that determine isotype-specific differences in complement activation. J. Exp. Med. 178, 661-667 (1993).

20. S. M. Canfield, S. L. Morrison, The binding affinity of human IgG for its high affinity Fc receptor is determined by multiple amino acids in the $\mathrm{CH} 2$ domain and is modulated by the hinge region. J. Exp. Med. 173 (1991).

21. T. E. Michaelsen et al., A mutant human IgG molecule with only one Clq binding site can activate complement and induce lysis of target cells. Eur. J. Immunol. 36, 129-138 (2006).

22. M. S. Kojouharova, I. G. Tsacheva, M. I. Tchorbadjieva, K. B. M. Reid, U. Kishore, Localization of ligand-binding sites on human C1q globular head region using recombinant globular head fragments and single-chain antibodies. Biochim. Biophys. Acta. 1652, 64-74 (2003).

23. I. Bally et al., Identification of the C1q-binding Sites of Human C1r and C1s: a refined three-dimensional model of the C1 complex of complement. J. Biol. Chem. 284, 19340-8 
(2009).

24. A. E. Phillips et al., Analogous interactions in initiating complexes of the classical and lectin pathways of complement. J. Immunol. 182, 7708-17 (2009).

25. V. U. Girija, A. R. Gingras, J. E. Marshall, R. Panchal, A. Sheikh, Structural basis of the $\mathrm{C} 1 \mathrm{q} / \mathrm{C} 1 \mathrm{~s}$ interaction and its central role in assembly of the $\mathrm{C} 1$ complex of complement activation. Proc. Natl. Acad. Sci. 110, 13916-20 (2013).

26. M. Budayova-Spano et al., Monomeric structures of the zymogen and active catalytic domain of complement protease $\mathrm{c} 1 \mathrm{r}$ : further insights into the $\mathrm{c} 1$ activation mechanism. Struct. London Engl. 1993. 10, 1509-1519 (2002).

27. M. Budayova-Spano et al., The crystal structure of the zymogen catalytic domain of complement protease $\mathrm{C} 1 \mathrm{r}$ reveals that a disruptive mechanical stress is required to trigger activation of the C1 complex. Eur. Mol. Biol. Organ. J. 21, 231-239 (2002).

28. A. J. Perry et al., A molecular switch governs the interaction between the human complement protease $\mathrm{C} 1$ s and its substrate, complement C4. J. Biol. Chem. 288, 15821$15829(2013)$

29. A. R. Gingras et al., Structural basis of mannan-binding lectin recognition by its associated serine protease MASP-1: implications for complement activation. Structure. 19, 1635-43 (2011).

30. H. Feinberg et al., Crystal structure of the CUB1-EGF-CUB2 region of mannose-binding protein associated serine protease-2. EMBO J. 22, 2348-59 (2003).

31. S. A. Mortensen et al., Structure and activation of $\mathrm{C} 1$, the complex initiating the classical pathway of the complement cascade. Proc. Natl. Acad. Sci. 114, 986-991 (2017).

32. S. E. Degn et al., Complement activation by ligand-driven juxtaposition of discrete pattern 
recognition complexes. Proc. Natl. Acad. Sci. 111, 13445-13450 (2014). 


\section{Acknowledgements}

Electron density maps are deposited in the EMBD. We gratefully acknowledge helpful discussions with Laura van Bezouwen, Dimphna Meijer, Friedrich Förster (Utrecht, Netherlands) and Sjors Scheres (Cambridge, UK) and technical assistance from Chris Schneijdenberg and Hans Meeldijk (EM-square, Utrecht). This research was supported by grants from the Netherlands Organization for Scientific Research (NWO) (project numbers CW 714.013.002, 700.57.010 and STW 13711), the Institute of Chemical Immunology (NWO 024.002.009) and the European Research Council (project 233229). This work was supported by the Netherlands Centre for Electron Nanoscopy (NeCEN), Leiden (NWO 175.010.2009.001).

\section{Conflict of interests}

JS, FJB, BJdK, RNdJ and PWHIP are inventors on patent applications related to complement activation by therapeutic antibodies. JS, FJB,BJdK and RNdJ are Genmab employees and own Genmab warrants and/or stock. PWHIP owns Genmab stock.

\section{Author contributions}

DU generated and purified mutant $\mathrm{C} 1 \mathrm{r}$ and $\mathrm{C} 1 \mathrm{~s}$, generated $\mathrm{C} 1-\mathrm{IgG} 1_{6}$, prepared grids, collected single-particle micrographs and processed data. DU and PG analysed single-particle data. RNdJ and DU designed $\mathrm{Ab}$ mutants and $\mathrm{BJdK}$ performed $\mathrm{CDC}$ and $\mathrm{CD} 20$ binding assays. SCH, RIK, AJK, and THS prepared liposomes and grids, and collected, processed and analysed tomography data. DU, RNdJ, THS, PWHIP and PG wrote the manuscript. All authors commented on manuscript. PWHIP and PG conceived the project. 

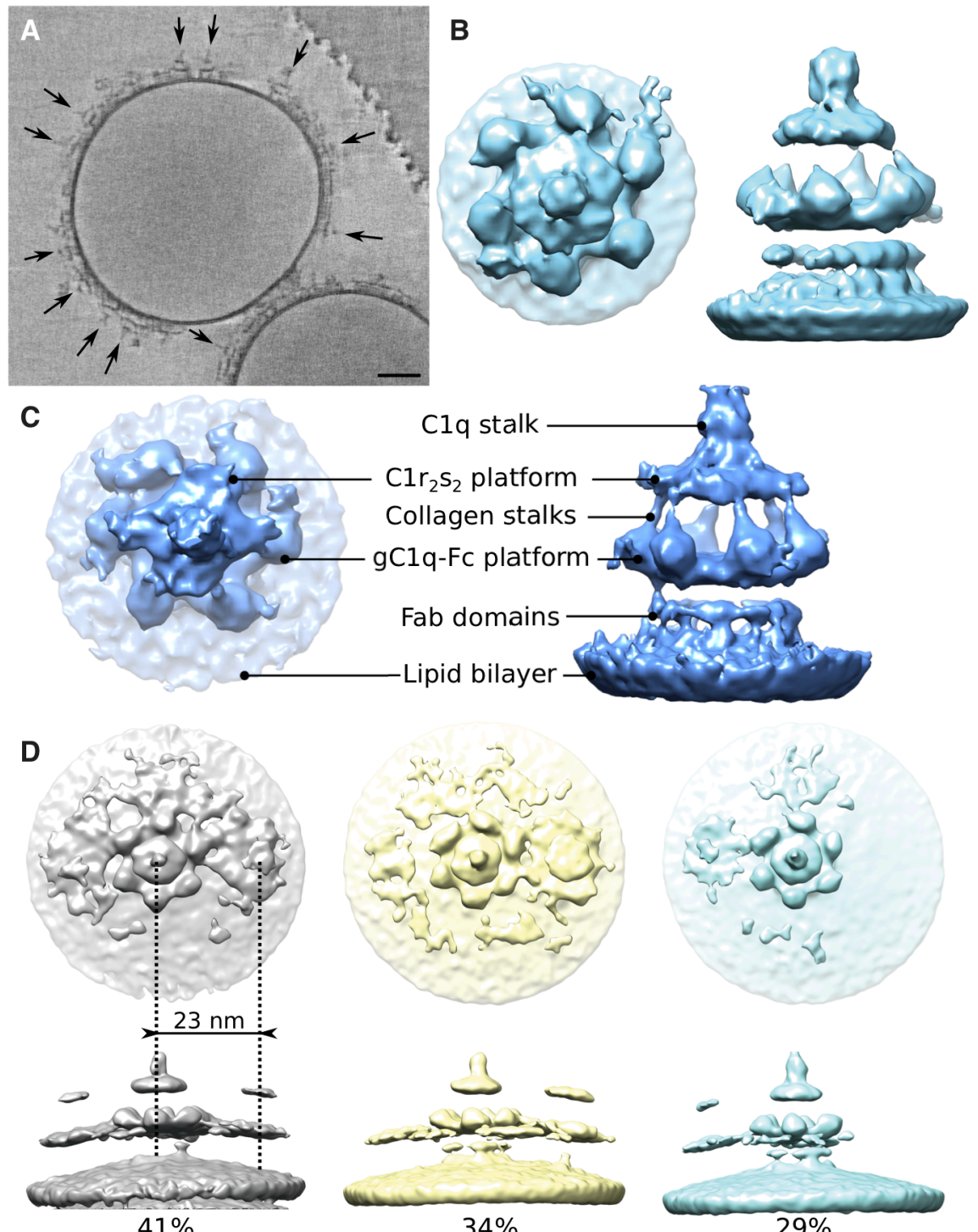

$41 \%$
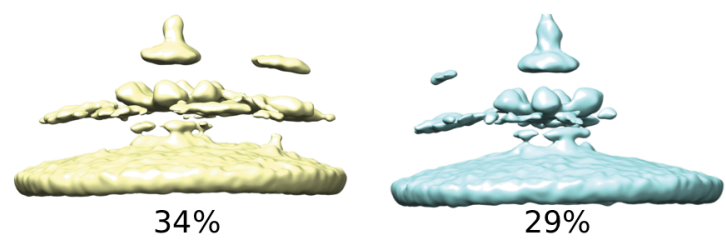

Fig. 1. C1-mAb complexes observed on liposomes by tomography.

(A) A $10 \mathrm{~nm}$-thick slice through a dual-axis tomogram showing $\mathrm{C} 1$ complexes (arrows) bound to surface-associated antibody complexes. Scale bar indicates $20 \mathrm{~nm}$. (B) Reconstruction of a single C1-IgG1 complex shown from the top (left) and side (right) at $25 \AA$ resolution. (C) Focused alignment and classification of the complexes excluding the membrane and Fab regions (see also Fig. S2E for masks used in focused reconstructions) revealed density from the $\mathrm{C}_{1} \mathrm{r}_{2} \mathrm{~s}_{2}$ platform extending out either side of the C1q stalk. (D) Neighboring C1-mAb complexes from larger subvolumes showing a common spacing of $23 \mathrm{~nm}$ between complexes, as measured from centers of 
IgG1 platforms. All volumes were filtered to $25 \mathrm{~nm}$ resolution, masked and disconnected densities with volumes less than $5 \mathrm{~nm}^{3}$ were removed for clarity. 


\begin{tabular}{|c|c|c|c|c|c|c|c|c|c|}
\hline A 4 & $\$$ & $\because 8$ & $\%$ & \% & $\ddot{*}$ & 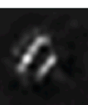 & $B$ & $\ddot{*}$ & 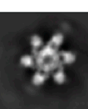 \\
\hline 1 & 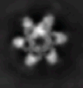 & 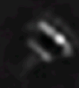 & $\nLeftarrow$ & $\$ 8$ & $\%$ & $\%$ & $\$$ & 26 & 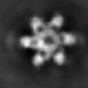 \\
\hline$\%$ & 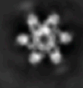 & a & $\nLeftarrow$ & $\%$ & 6 & C) & $\not \gamma$ & 86 & 17 \\
\hline 7 & r. & 7 & () & $\approx 8$ & $\mathscr{H}$ & $\Rightarrow$ & $\because 8$ & 7 & $\$$ \\
\hline
\end{tabular}

B
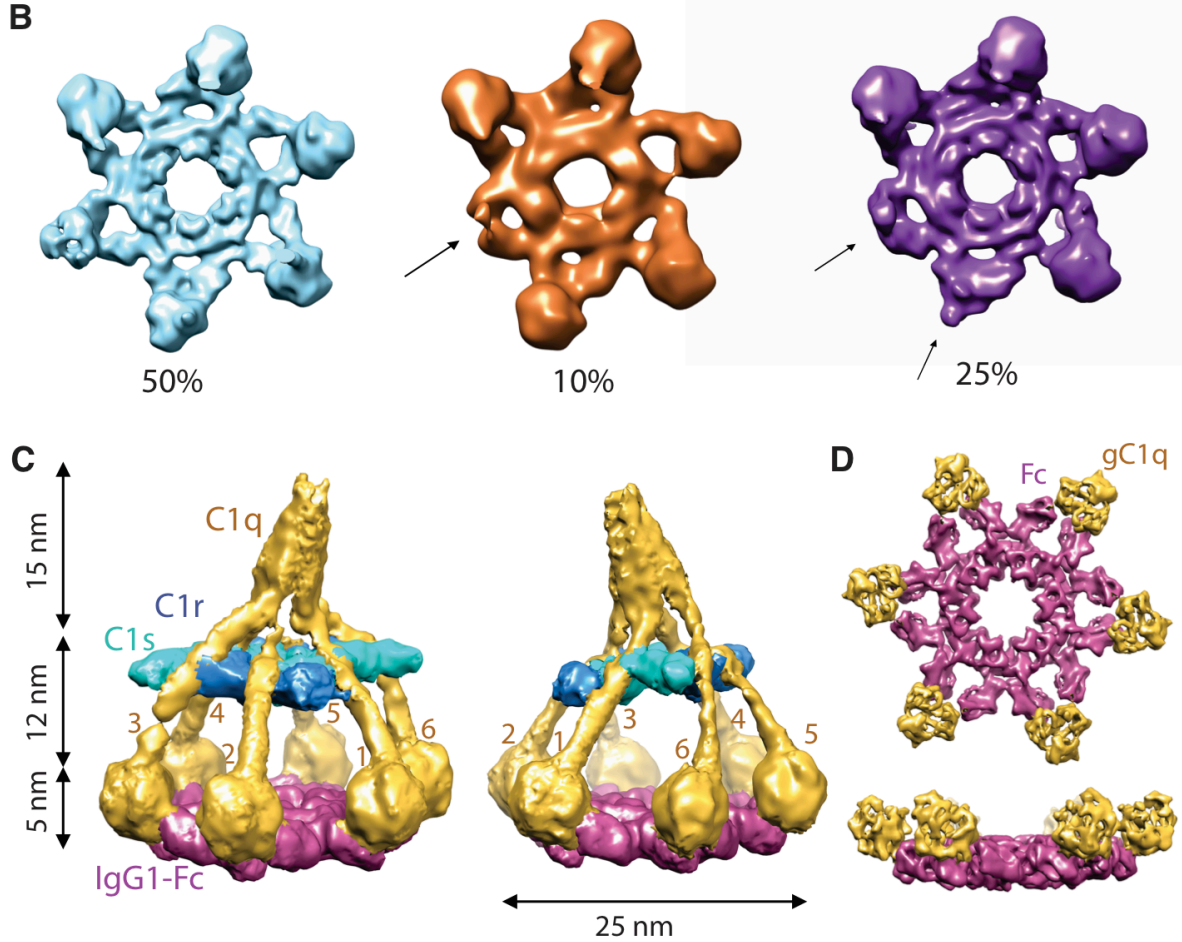

Fig. 2. Soluble C1-IgG1 6 complexes display heterogeneous structures.

(A) Representative 2D class averages. Colored boxes indicate three classes corresponding to main 3D classes, as shown below. Scale bar represents $25 \mathrm{~nm}$. (B) Main 3D classes after focused 3D classification and 3D refinement, showing the 'bottom platform' segment of the reconstructions indicating heterogeneities (highlighted by arrows). Percentage of particles in each class are indicated. Particle colors correspond to the color of the boxes in panel A. (C) 3D reconstructions after post-processing of the major class, showing two side views (left and middle). Densities have been coloured indicating density for Clq (yellow; with collagen arms 
and $\mathrm{gClq}$ units numbered 1 through 6), $\mathrm{C} 1 \mathrm{r}$ and $\mathrm{C} 1 \mathrm{~s}$ (blue and magenta, resp.) and $\mathrm{IgG1}-\mathrm{Fc}$ regions (pink). (D) Top and side views of the bottom platform after six-fold averaging (right top and bottom, respectively). 

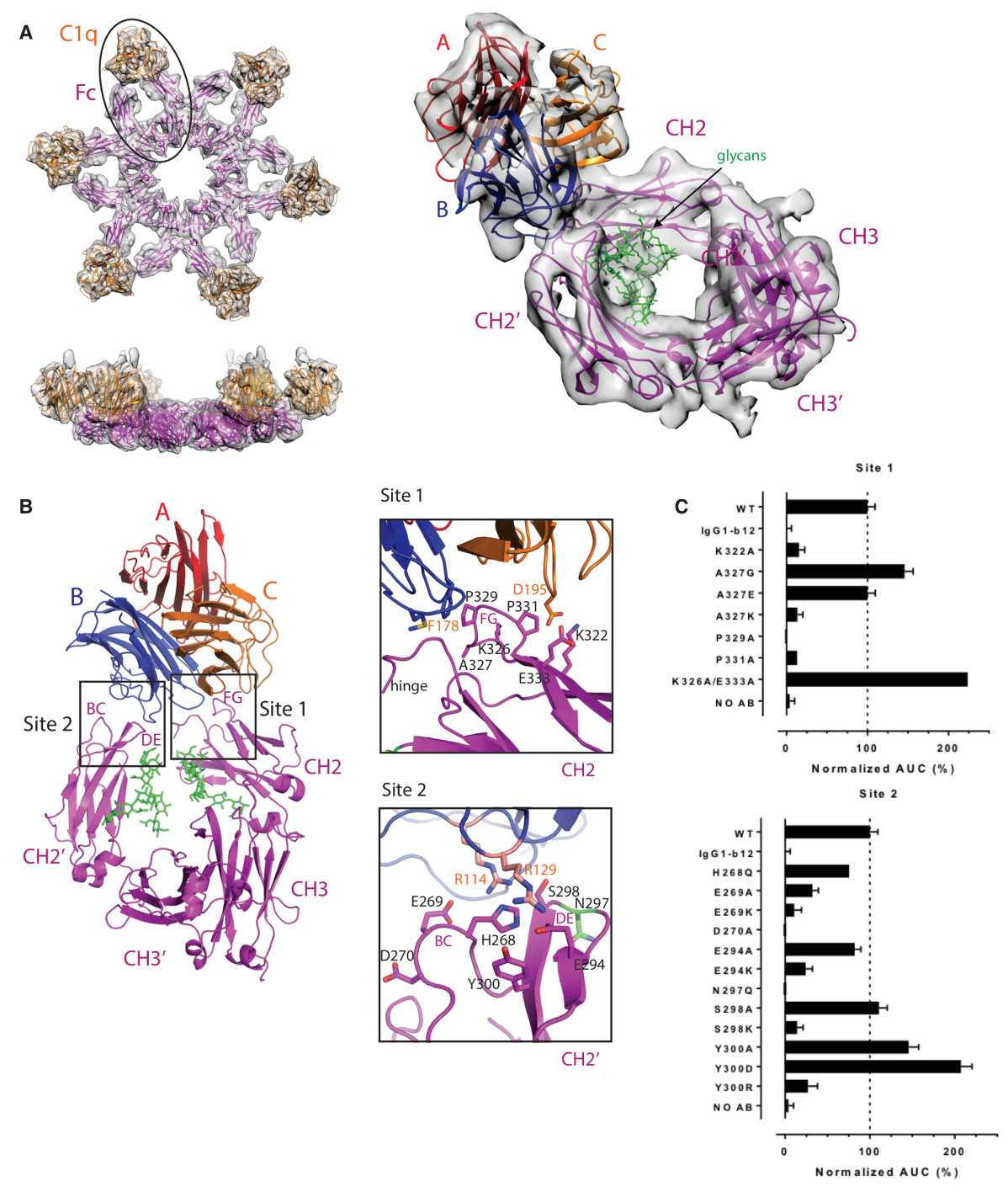

Fig. 3. Fc-gC1q interactions in C1-IgG16.

(A) Structural models of Fc regions (magenta) and gClq headpieces (orange) fitted into the density, top and side view of the Fc-gClq hexamer (left) and zoom in of a gClq trimeric with C1q-A, B and C domains (red, blue, orange resp.) and an Fc dimer with $\mathrm{CH} 2-\mathrm{CH} 3$ and $\mathrm{CH} 2$ 'CH3' (right), with Fc glycans shown in green. (B) gC1q-Fc interaction site 1 and site 2 are shown indicated by boxes, with interacting loops FG (site 1), and BC and DE (site 2) labelled (left). Zoom in of interaction sites 1 and 2 (right) with key interacting residues shown in stick representation and labelled. (C) Complement dependent cytotoxicity assays of Raji cells 
opsonized with wild-type (WT) and mutated CD20 mAb IgG1-7D8 (n=3) exposed to C1qdeficient serum to which a titration of $1 \mathrm{ng} / \mathrm{mL}$ to $60 \mu \mathrm{g} / \mathrm{mL} \mathrm{C1q}$ was added. Cell lysis was assessed by flow cytometry using propidium iodide staining. Bars show the average area under the curve (AUC) for this dose response normalized against the AUC obtained with the unmutated WT IgG1-7D8 set to 100\% NO AB: control reactions without IgG1 added. 

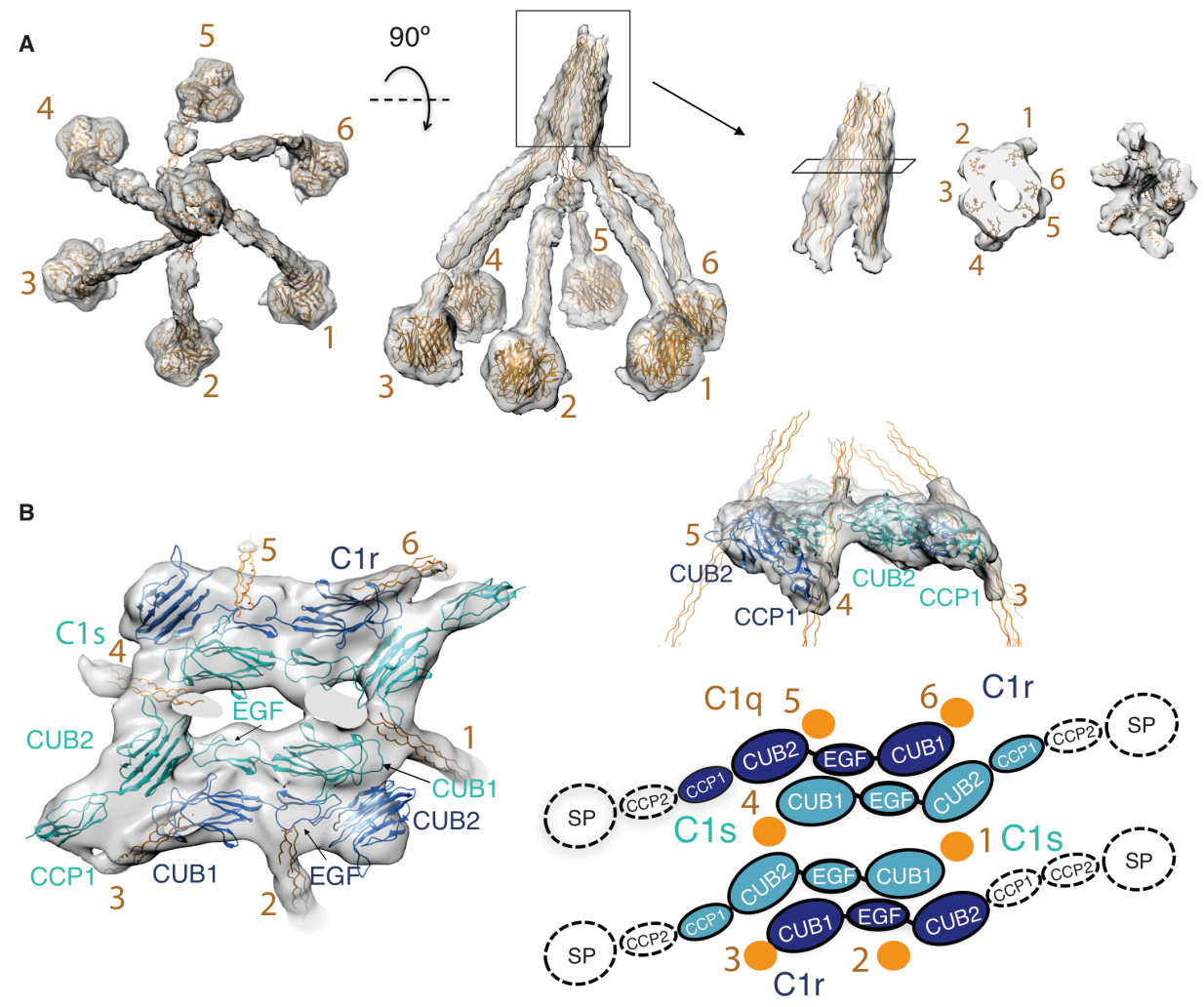

C

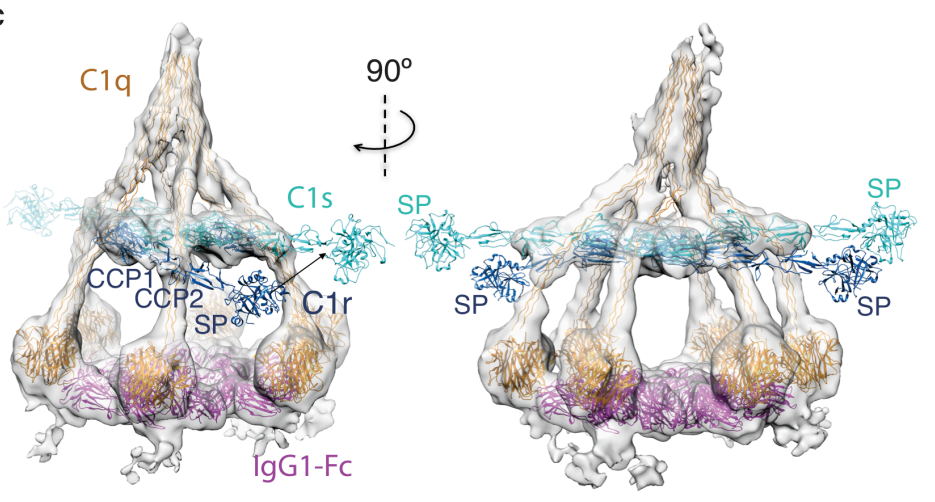

Fig. 4. Structural model of C1 fitted into C1-IgG1 6 density.

(A) Model for C1q-A, B and C hexamer indicating collagen-like segments forming a N-terminal stalk region, six collagen-like triple helices and C-terminal trimeric gC1q modules. Shown are top and side views (left and middle) of C1q and side, sliced-through top and bottom view ( $3^{\text {rd }}$ column left to right) of the C1q stalk region. Numbering of each C1q arm as in Fig. 2. (B) Model for C1r and C1s hetero-tetramer showing C1r CUB1-EGF-CUB2 (blue) and C1s CUB1-EGFCUB2-CCP1 domains (cyan). Shown are top view (left) and side view at lower contour level 
(top right), with the latter revealing density for the $\mathrm{CCP} 1$ domain of $\mathrm{C} 1 \mathrm{r}$. A cartoon representation of the domain arrangement is shown for clarity (bottom right). (C) Overall C1IgG1 6 models in density. CCP2-SP domains lacking density have been added, using orientations derived from crystal structures. 


\section{Supplementary Materials contents:}

\section{Materials and Methods}

Figs. S1 to S7

Tables S1

References

\section{Materials and Methods}

\section{Expression and Purification of the Proteins}

Plasma-purified human C1q was purchased from Complement Technology, Inc. (Tyler, TX, USA). IgG1-RGY variant (E345R, E430G, and S440Y), IgG1-b12 and IgG1-7D8 were described previously $(5,15,33)$. IgG1-DNP consisted of the murine variable domain of antibody G2a2 fused to a human IgG1 constant domain (34). IgG1-DNP and IgG1-7D8 antibody variants were created by gene synthesis of codon optimized open reading frames (Geneart Thermo Scientific, Regensburg, Germany), subcloned into pcDNA3.3-derived expression vectors. All antibodies were expressed, purified and characterized as described (14). C1q-depleted serum was obtained from Quidel (San Diego, CA).

C1r (S654A) and C1s (S632A) mutants were generated using the Quikchange mutagenesis kit (Agilent Genomics, Santa Clara, CA) on TOPO clones inserted in pCR8 vectors (Thermo Fisher Scientific, Waltham, MA). All C1r and C1s constructs were sub-cloned into mammalian expression vectors carrying either a hexa-histidine tag or StrepII3-tag provided by U-Protein Express BV (U-PE), Utrecht, The Netherlands. C1r and C1s constructs were coexpressed in N-acetylglucosaminyltransferase I (GnTI)-deficient human embryonic kidney 293 cells that stably express Epstein-Bar virus nuclear antigen EBNA1 (HEK293-ES supplied by U-Protein Express BV). The supernatant was harvested on the $5^{\text {th }}$ day of the expression, concentrated and dia-filtrated using a Quixstand system (GE Healthcare, Chicago, IL). C1 $\mathrm{r}_{2} \mathrm{~s}_{2}$ was purified by Ni-NTA affinity chromatography (GE Healthcare) followed by a size-exclusion chromatography step on a Superdex 200 column (GE Healthcare). $\mathrm{C} 1-\mathrm{IgG1}_{6}$ complexes were prepared by mixing $\mathrm{C}_{2} \mathrm{r}_{2} \mathrm{~s}_{2}, \mathrm{C} 1 \mathrm{q}$ and $\mathrm{IgG} 1-\mathrm{RGY}$ in 4:1:6 molar ratio. The complex was purified by gel filtration column BioSep $400015 \mathrm{ml}$ column.

\section{Cell culture}


Raji cells (human Burkitt's lymphoma) were obtained from the American Type Culture Collection (ATCC no. CLL-86; Rockville, MD) and cultured according to the supplied instructions. Cells were routinely tested for mycoplasma contamination.

\section{Tomography sample preparation and data collection}

Liposomes were prepared from dried films of cholesterol, DMPC, DMPG and cap-DNP-PE (all sourced from Avanti Polar Lipids, Alabaster, AL) at a molar ratio of 100:90:10:1, respectively. Films were hydrated in buffer (50 mM HEPES, pH 8.0, $145 \mathrm{mM} \mathrm{NaCl}$, and 3 $\mathrm{mM} \mathrm{CaCl} 2$ ) at $37^{\circ} \mathrm{C}$ for 4 hours and extruded through $200 \mathrm{~nm}$ pore filters at $45^{\circ} \mathrm{C}$. Liposomes, IgG1-DNP, and plasma-purified human C1 complex (Complement Technology Inc., Tyler, TX) were mixed on ice at final concentrations of $1.3 \mathrm{mg} / \mathrm{ml}, 0.025 \mathrm{mg} / \mathrm{ml}$ and $0.017 \mathrm{mg} / \mathrm{ml}$, respectively, then brought to room temperature and allowed to equilibrate for approximately 30 minutes. Gold fiducials coated in bovine serum albumin (Aurion, Wageningen, The Netherlands) were added to the samples immediately before $3 \mu 1$ of the mixture was applied to freshly glow-discharged Quantifoil R2/1 300 mesh holey carbon grids in the chamber of a Leica EM-GP plunge-freezer (Leica, Germany) set at 96\% humidity and $21^{\circ} \mathrm{C}$. Grids were blotted for $1 \mathrm{sec}$ and plunged into liquid ethane.

Tomograms were collected at NeCEN (Leiden, The Netherlands) on a Titan Krios (Thermo Fisher Scientific) operating at $300 \mathrm{kV}$ equipped with a phase plate (Thermo Fisher Scientific) heated to $225^{\circ} \mathrm{C}$. Zero-energy-loss images were acquired using the software program FEI Tomography 4 (Thermo Fisher Scientific), using a GIF-Quantum LS energy filter (Gatan, Pleasanton, CA) with a slit width of $20 \mathrm{eV}$. A K2 Summit direct electron detector (Gatan) was used in counting mode at a nominal magnification of $53,000 \times$ for a pixel size of $2.69 \AA$ at the sample. Tomographic tilt series were collected from $\pm 50^{\circ}$ in $2^{\circ}$ increments with a dose rate of 0.9 electrons per $\AA^{2}$ per sec for a total dose of 60 electrons per $\AA^{2}$ per tilt axis (dualaxis tomograms received a total dose 120 electrons per $\AA^{2}$ ). Exposures of 0.9 seconds were dose-fractionated into 6 movie frames per tilt angle. Before each tilt series, the Volta phase plate was advanced and conditioned for 5 minutes to generate an approximate phase shift of $90^{\circ}$. The phase plate was also conditioned for an additional $5 \mathrm{~s}$ between every fifth tilt image. Defocus values were set to $-0.3 \mu \mathrm{m}$ after tilting and before each image acquisition, which is sufficient to maintain negative phase contrast up to $\sim 19 \AA$ (35).

\section{Tomography reconstruction and sub-volume averaging}

Raw frames were aligned using MotionCorr (36) and tomograms were reconstructed using 
IMOD (37) after binning the aligned frames by 2 (final pixel size of $5.38 \AA$ ). Single- and dual-axis tomograms were processed using the same workflow for the reconstruction of each axis. Axes for dual-axis tomograms were combined using IMOD. The surfaces of liposomes were manually segmented from simultaneous iterative reconstruction technique (SIRT) reconstructions using Dynamo (38) and equally spaced boxes across the surface were extracted from weighted back-projection reconstructions for further processing. Dynamo tracks the orientation for each particle, and thereby allows for Fourier-space masks to be constructed for each sub-volume to account for missing information caused by selected tilt geometries (38). An initial model was generated by manually aligning 107 sub-volumes from the dual-axis tomograms and low pass filtered to $6 \mathrm{~nm}$ (Fig. S2C). White noise was added using EMAN2 (39) to five copies of the filtered initial model, which were then used for the classification and alignment of all the dual-axis sub-volumes. The best average from the dualaxis alignments was again low pass filtered to $6 \mathrm{~nm}$ and four noisy copies used for the classification and alignment of the single-axis sub-volumes. Initial rounds of classification for bare or decorated membrane surfaces were performed using data further binned by 2 (binned by 4 from the raw images) and low pass filtered, using the membrane orientation as a constraint on the alignment parameters $\left( \pm 30^{\circ}\right.$ maximum deviation in the sub-volume's inclination from the starting orientation and full freedom to rotate in the azimuthal direction). If the aligned sub-volumes were not separated by at least $10 \mathrm{~nm}$ in the original tomogram, the sub-volume with the lower correlation score was discarded from further processing. From classes that were not bare membrane, sub-volumes containing $\mathrm{C} 1$ complexes (often mixed with antibody-alone sub-volumes) were manually selected and the correct orientation verified from individual sub-volume projections. These coordinates were used to re-extract the subvolumes from weighted-back projection tomograms. The sub-volumes were then subjected to a global orientation search again using binned and low-pass filtered data for the initial rounds and refined following gold-standard procedures. Sub-volumes that did not align correctly were again manually discarded by inspecting projections of the sub-volumes after the orientation determined by the alignment procedure was applied. The $\mathrm{C} 1-\mathrm{mAb}$ complex shape and the membrane provide obvious features to verify the alignment. The final stack of verified $\mathrm{C} 1-\mathrm{mAb}$ sub-volumes was then subjected to further classification using the ellipsoidal mask shown in Fig. S2E to generate the classes shown in Fig. S2C. A box size of 128 pixels cubed was used for the initial alignment and classification steps of the dual-tilt tomograms. For the focussed alignment and classification shown in Fig. 1C, particles were aligned using the cylindrical mask shown in Fig. S2E and a box size of 84 pixels. A box size 
of 168 was used to investigate the neighboring $\mathrm{C} 1-\mathrm{mAb}$ complexes using a biased ellipsoid mask that fitted three mAb-C1 complexes in a line (Fig. S2E). The nearest neighbor distances were determined by computing the distance between aligned sub-volumes within each tomogram. The $\mathrm{x}-, \mathrm{y}-$ and $\mathrm{z}$-shifts were added to the original coordinates of the sub-volume to calculate the $\mathrm{C} 1$ complex centre. The center-to-center distance for all particles was calculated and the minimum pairwise value was taken as the nearest neighbour distance shown in Fig. S2F. The final number of sub-volumes was 564 dual-axis and 3,190 single-axis. The final number of particles that contributed to the average shown in Fig. 1B is 1,660.

\section{Sample preparation and data collection for single-particle cryo-EM}

A volume of $3 \mu \mathrm{l}$ of purified $\mathrm{C} 1-\mathrm{IgG1}_{6}$ at a concentration of $0.2 \mathrm{mg} / \mathrm{ml}$ was applied to freshly glow discharged R1.2/1.3 holey carbon grids (Quantifoil). The grids were blotted for $1 \mathrm{~s}$ at 99\% humidity in a Vitrobot plunge-freezer (Thermo Fisher Scientific). Cryo-EM images were collected at EM-square (Utrecht, The Netherlands) on a Talos Arctica (Thermo Fisher Scientific) operating at $200 \mathrm{kV}$ equipped with a Falcon II direct detector (Thermo Fisher Scientific) operating in movie mode. Images were recorded manually at a nominal magnification of $42,000 \times$ yielding a pixel size at the specimen of $2.27 \AA$. Using the EPU software (Thermo Fisher Scientific), 3,493 micrographs were recorded. Movies were collected for $3 \mathrm{~s}$ with a total of 52 frames with a calibrated dose of $10.4 \mathrm{e}^{-} / \AA^{2}$ per frame, a total dose of $\sim 53 \mathrm{e}-/ \AA^{2}$, and at defocus values between -1.8 and $-3 \mu \mathrm{m}$. An example micrograph is shown in Fig. S4A.

\section{Single Particle Analysis}

Movie stacks were aligned using MotionCorr (36). CTF parameters were defined using Ctffind4 (40). A total of 282,150 particles were picked using particle-picking software from Relion using 2D class averages of 2500 manually picked particles (41); see Fig. S4C for a schematic representation of the workflow. The particles were aligned and classified iteratively by $2 \mathrm{D}$ classification according to their $2 \mathrm{D}$ projection views using the software package Relion (42). $\sim 168,341$ particles were selected from classes with top projections of 6 $\mathrm{gClq}$ domains bound to Fc platform and side views with visible collagen arms for ab initio 3D reconstruction in Cryosparc (43). Second set of 65,768 particles were selected from classes with top projections of 4 or $5 \mathrm{gClq}$ domains bound to $\mathrm{Fc}$ platform and the remaining side views. From the first particle set (left column in Fig.S4C), an unbiased 3D reconstruction without reference and mask yielded a class with $\sim 79,120$ particles which was selected as an 
initial model. The map was low-pass filtered and used as a reference for 3D classification on the $\mathrm{gClq}-\mathrm{Fc}$ platform. A soft mask in Relion around $\mathrm{gClq}-\mathrm{Fc}$ platform was generated by extending 3 pixels and softening the edge by further 10 pixels. Focused classification using this mask yielded five classes with a resolution up to $15 \AA$. 3D refinement of a selection of 126,372 particles in Relion resulted in a $14 \AA$ map. Two masks were generated using this map around the full C1-IgG6 complex (extended by 3 pixels and softening by 10 pixels) and around gC1q-Fc platform (extended by 2 pixels and softening by 20 pixels). The initial selected class of 79,120 particles was used for 3D refinement in Cryosparc (homogenous refinement option) with the $14 \AA$ map used as a reference and the mask around full $\mathrm{C} 1-\operatorname{IgG} 1_{6}$ complex. After 3D refinement in Cryosparc and postprocessing in Relion, the resolution was $10 \AA$. 3D refinement applying C6 symmetry with the mask around gC1q-Fc platform and subsequent post-processing resulted in $7.3 \AA$ map. The resolutions were estimated by Fourier shell correlation between two half maps (each calculated independently) as a function of resolution and using the gold-standard FSC $=0.143$ criterion. For classification and refinement, a box size of 224 pixels was used. Particle-polishing procedure implemented in Relion software did not improve the resolution (data not shown). The second set of particles (right column in Fig.S4C) were used for 3D classsification using a mask around gC1q-Fc platform. 44,121 particles showing $4 \mathrm{gClq}$ domains abound to $\mathrm{Fc}$ platform was reconstructed by $3 \mathrm{D}$ refinement and postprocessing. 12,781 particles showing $5 \mathrm{gC} 1 \mathrm{q}$ domains bound to $\mathrm{Fc}$ platform was reconstructed by $3 \mathrm{D}$ refinement and postprocessing.

\section{Modeling C1 structure into density map}

Crystal structures were fitted into densities using the "Fit in Map" routine from Chimera (44) Fc CH2, CH3, CH2' and CH3' domains were taken from the IgG1 b12 structure (pdb-code $1 \mathrm{HZH})(15)$ and fitted independently into the density map obtained at 7.3- $\AA$ resolution. $\mathrm{gC} 1 \mathrm{q}$ globular heads (1PK6) (16) were fitted in the same map in three orientations for the C1q-A, B and $\mathrm{C}$ domains and the orientation with the highest correlation coefficient (0.95) calculated by Chimera was selected. The serine proteases $\mathrm{C} 1 \mathrm{r}$ and $\mathrm{C} 1 \mathrm{~s}$ were fit into the map using previously modelled arrangements of $\mathrm{C}_{1} \mathrm{r}_{2} \mathrm{~s}_{2}$ bound to $\mathrm{C} 1 \mathrm{q}(23-25)$ see Fig. S7A. The heterodimer C1rs started from the C1s homo-dimer structure of CUB1-EGF-CUB2 (4LMF) (25). One C1s copy was modified into C1r CUB1-EGF-CUB2 using homology modeling (SWISSMODEL) (45) to generate the initial C1rs model. C1r and C1s CUB1 interactions with collagen (i.e. with $\mathrm{C} 1 \mathrm{q}$ arm 3 and 1 resp.) were modeled using the orientations found in the crystal structure of C1s CUB1-EGF-CUB2 bound to a collagen peptide (4LOR) (25). C1r 
CUB2 interaction with collagen of C1q arm 2 was modeled using the crystal structure of MASP CUB2 bound to collagen peptide (3POB) (29). This model of the C1rs hetero-dimer, C1r CUB1-EGF-CUB2 and C1s CUB1-EGF-CUB2, was placed into the density map obtained at $10-\AA \AA$ resolution and adjusted to fit the density (Fig. S7A). Available densities for $\mathrm{C} 1 \mathrm{r}$ and $\mathrm{C} 1 \mathrm{~s} \mathrm{CCP} 1$ domains were modeled using the C1s CUB2-CCP1 structure (4LOS) (25). The model of C1rs CUB1-EGF-CUB2-CCP1 heterodimer was duplicated and rotated by $180^{\circ}$ to model the other half of the density and minor adjustments were made to fit the density. Finally, we predicted the positions for the CCP2-SP domains of $\mathrm{C} 1 \mathrm{r}$ and $\mathrm{C} 1 \mathrm{~s}$, for which no densities were observed in the map. Starting from the positions and orientations of CUB-CCP1 domains, the model was extended using CCP1-CCP2-SP from the structure of zymogen C1r (1GPZ and 1MD7) $(26,27)$ and zymogen C1s (4J1Y) $(28)$, and aligning them according to the arrangement observed in the structure of the C1s CUB2-CCP1-CCP2 fragment (4LOT) (25). The cross-correlation coefficient of $\mathrm{C} 1-\mathrm{IgG} 1_{6}$ model and the final map was 0.78 and $\mathrm{gClq}-\mathrm{Fc}$ and the bottom platform was 0.65 according to the scoring assessment tool of TEMPy (46).

\section{Complement-dependent cytotoxicity assay}

Raji cells were washed twice with PBS and subsequently re-suspended in RPMI 1640 medium (containing 0.1\% BSA and Pen/Strep). Cells were added to a 96-well plate (1E+05 cells/well) followed by addition of $\mathrm{mAbs}$ at a concentration of $10 \mu \mathrm{g} / \mathrm{mL}$ and then incubated for 15 minutes (RT). Subsequently, a concentration series of $1 \mathrm{ng} / \mathrm{mL}$ to $60 \mathrm{ug} / \mathrm{mL} \mathrm{C1q}$ protein (Quidel, A400) was added, and C1q-deficient serum (Quidel, A509) was added to a final concentration of $20 \%$ as a source of complement. After incubation for 45 minutes at $37^{\circ} \mathrm{C}$, cells were immediately placed on ice. After centrifugation, supernatant was discarded and cells were re-suspended in BSA-PBS containing $1 \mu \mathrm{g} / \mathrm{mL}$ PI. The fraction of PI $(+)$ cells $(100 \% *(\mathrm{PI}(+)$ cells/total cells) was determined using an iQue Screener flow cytometer (Intellicyt, Albuquerque, NM). The area under the curve (AUC) of three experimental replicates was calculated using a log transformed concentration axis with GraphPad Prism 7.02 and normalized relative to cell lysis measured for wild type IgG1-7D8 (WT) set at 100\% and the nonbinding control IgG1-b12 set at $0 \%$. AUC values were collected for IgG1-7D8 antibody variants in two independent series of three experimental replicates. Mean AUC (normalized per series) and standard deviation (SD) were determined and are presented in figures 3 and S6. One-way ANOVA of mean AUC was followed by Dunnett's Multiple Comparison Posthoc Test using GraphPad Prism 7.02. A p value $<0.05$ indicates that CDC 
obtained with the mutant is different from that obtained with WT 7D8. Data is summarized in supplementary table S1. Without C1q added, background cell lysis by $10 \mu \mathrm{g} / \mathrm{mL}$ IgG1-b12 or IgG1-7D8 was similar. 

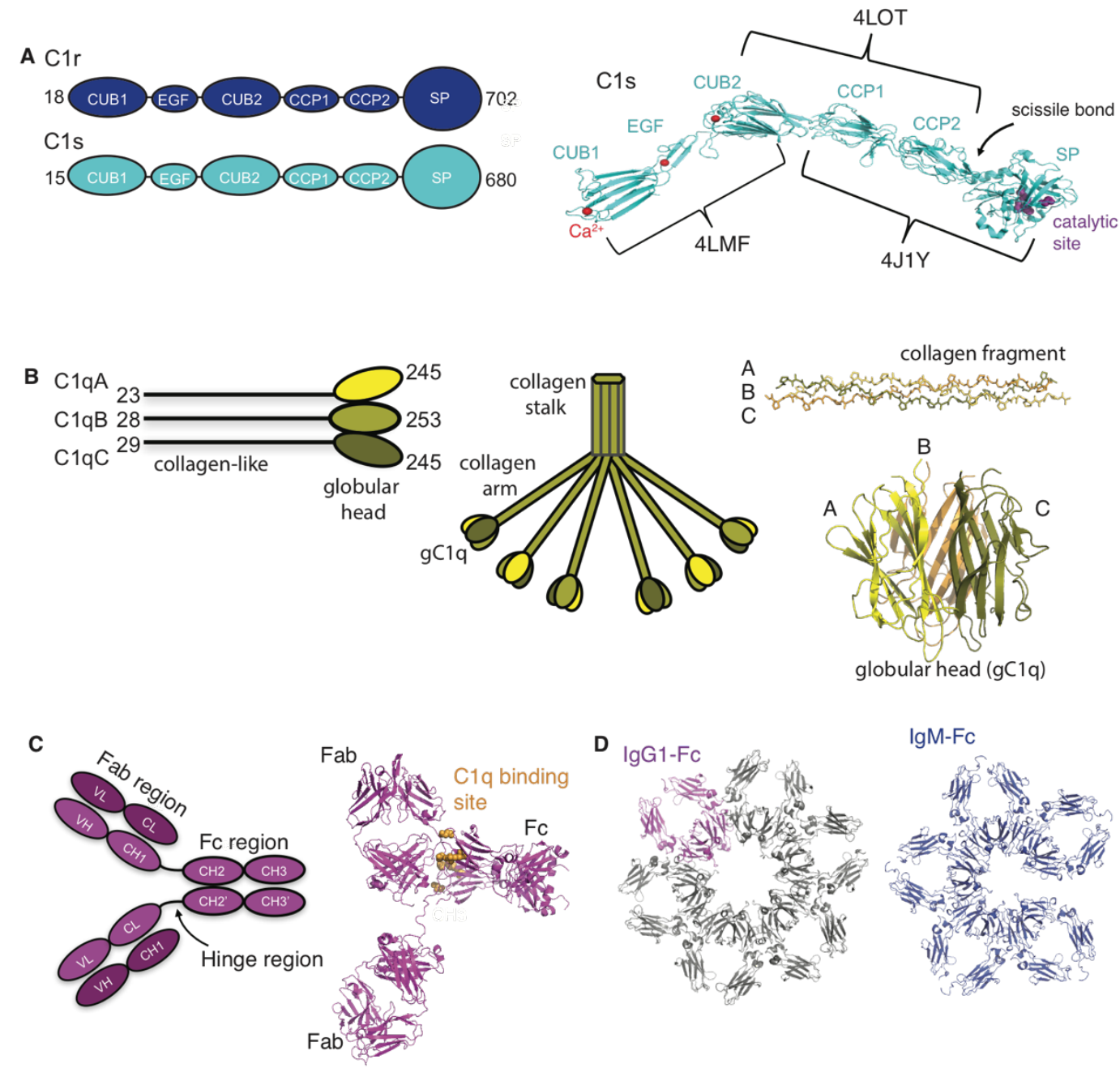

\section{Supplementary Fig. S1. Domain compositions and prior structural information.}

(A) Domain compositions of $\mathrm{C} 1 \mathrm{r}$ and $\mathrm{C} 1 \mathrm{~s}$, indicating the first 'Complement $\mathrm{C} 1 \mathrm{r} / \mathrm{C} 1 \mathrm{~s}$, Uegf, Bmp1' (CUB1), epidermal growth factor-like (EGF), second CUB (CUB2), first complement-control-protein (CCP1), second CCP (CCP2), and serine-protease (SP) domains; and, composite structural model of $\mathrm{C} 1 \mathrm{~s}$ built up from overlapping crystal structures (with pdb-codes 4LMF, 4LOT and 4JIY, as indicated $(25,28))$. Generation of a composite model for $\mathrm{C} 1 \mathrm{r}$ yields a highly similar domain arrangement as that for $\mathrm{C} 1 \mathrm{~s}$ (not shown). Indicated are the three calcium-binding sites (by red calcium ion), scissile bond (arrow) and catalytic site (purple). (B) Composition of C1q, showing the hetero-trimer chains A, B and C, which form a collagen-like region and trimeric globular head (left). Six chain of C1q A, B and C form the overall Clq assembly (middle). A collagen triple helix model, (colored and labeled with as a hetero-trimer, mimicking the situation in $\mathrm{C} 1 \mathrm{q})$ and the crystal structure of $\mathrm{gC} 1 \mathrm{q}(1 \mathrm{PK} 6)(16)$. (C) Composition of an IgG molecule, showing light chain and variable constant domains (VL 
and $\mathrm{CL}$, resp.) and heavy chain variable, constant-1, 2 and 3 domains (VH, $\mathrm{CH} 1, \mathrm{CH} 2$ and $\mathrm{CH} 3$, resp.), antigen-binding fragment (Fab), Fc and hinge region. Shown are a cartoon (left) and crystal structure 1HZH (15). Residues involved in C1q binding are schematically shown by yellow dots. (D) Six-fold arrangements of IgG1 Fc domains as observed in the crystal packing contacts in pdb 1HZH. The hexamer is shown in black with the Fc domain of a single IgG molecules highlighted in red (left). Model of the Fc arrangement in a single pentameric IgM molecule (right) (47).

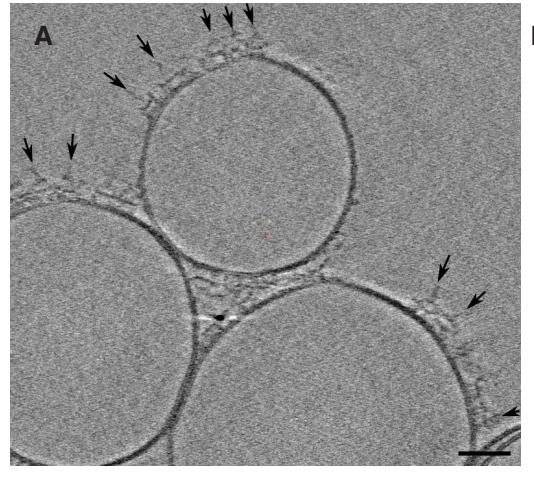

C

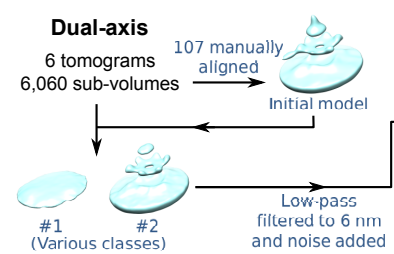

564 manually confirmed $\downarrow$ allgned sub-volumes

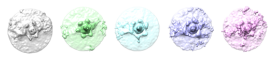

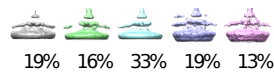

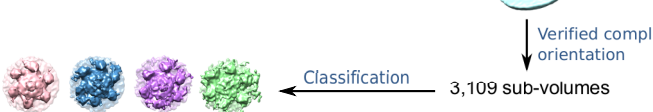

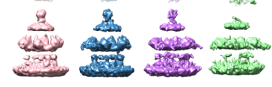

$\stackrel{34 \% \quad 26 \% \quad 20 \% \quad 19 \%}{\downarrow}$

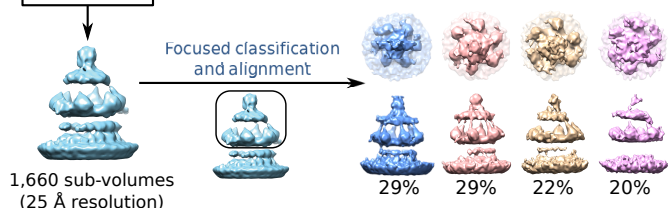

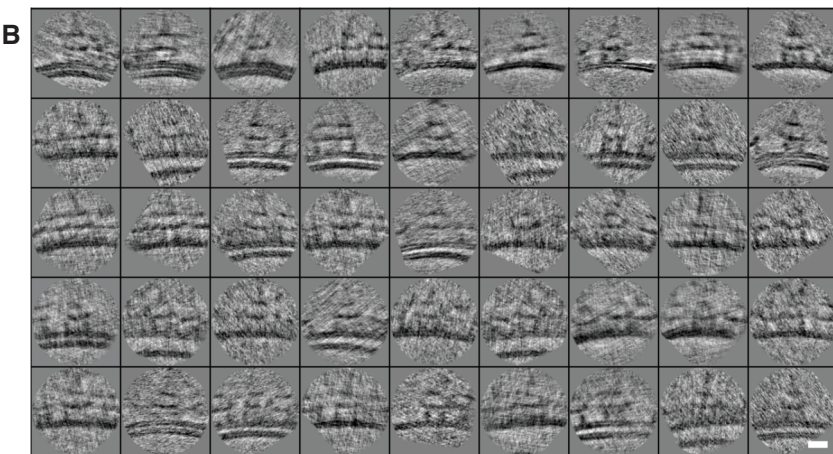

D

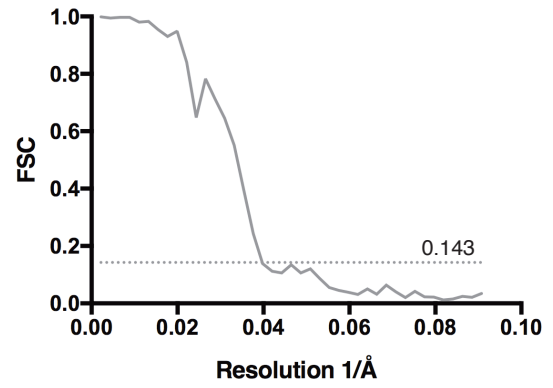

E

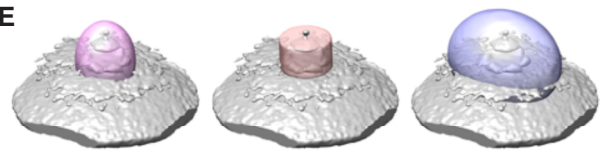

$\mathbf{F}$

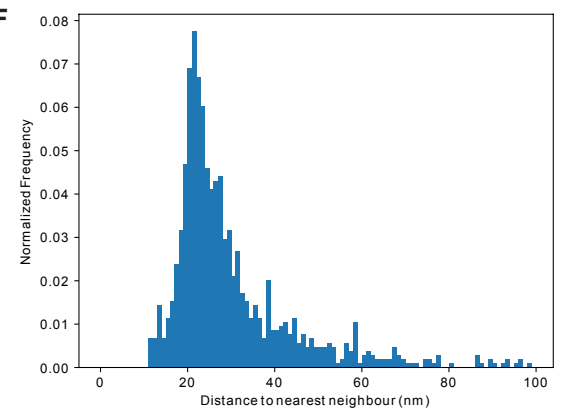

Supplementary Fig. S2. Cryo-EM tomography of C1 bound to IgG1 on liposomal 


\section{surfaces and image analysis workflow.}

(A) A $10.8 \mathrm{~nm}$-thick slice through a single-tilt tomogram of liposomes decorated with a chimeric anti-DNP IgG1 monoclonal antibody and C1 complexes. The scale bar indicates 20 nm. (B) Raw images of IgG1-C1 complexes from single-tilt tomograms after coarse alignment. The scale bar indicates $10 \mathrm{~nm}$. (C) Sub-volume alignment and classification workflow. A subset of 107 sub-volumes from dual-axis tomograms were manually aligned to generate an initial model. The initial model was used to align and classify the dual-axis subvolumes. The resulting average from the dual-axis data was used to process the single-axis sub-volumes. Due to the extensive flexibility, all assignments into the decorated class and final orientation of the aligned sub-volume were manually verified. Multi-reference refinement using five classes (dual-tilt) and four classes (single-tilt) revealed classes showing flexibility of the structure and consistent presence of six IgG1-Fc and gC1q domaincontaining densities. The percentage of sub-volumes assigned to each class is shown below the image. From the single-axis sub-volumes, two of these classes were nearly identical and contained the majority of particles (54\% total), which were subjected to a final alignment round, which yielded the density map shown in Fig. 1B. Focussed alignment and classification on the Fc domains and $\mathrm{C} 1$ complex yielded the reconstruction shown in Fig. 1C. (D) Fourier shell correlation (FSC) curve for the complete IgG1-C1 complex showing $\mathrm{FSC}_{0.143}=25 \AA$. (E) Masks used during sub-volume alignment and classification of complete mAb-C1 complexes (left), focussed refinement of the Fc-C1 complex (middle), and to investigate neighboring $\mathrm{mAb}-\mathrm{C} 1$ complexes (right). (F) Histogram showing the calculated distances between nearest neighbour mAb-C1-IgG1 complexes. 

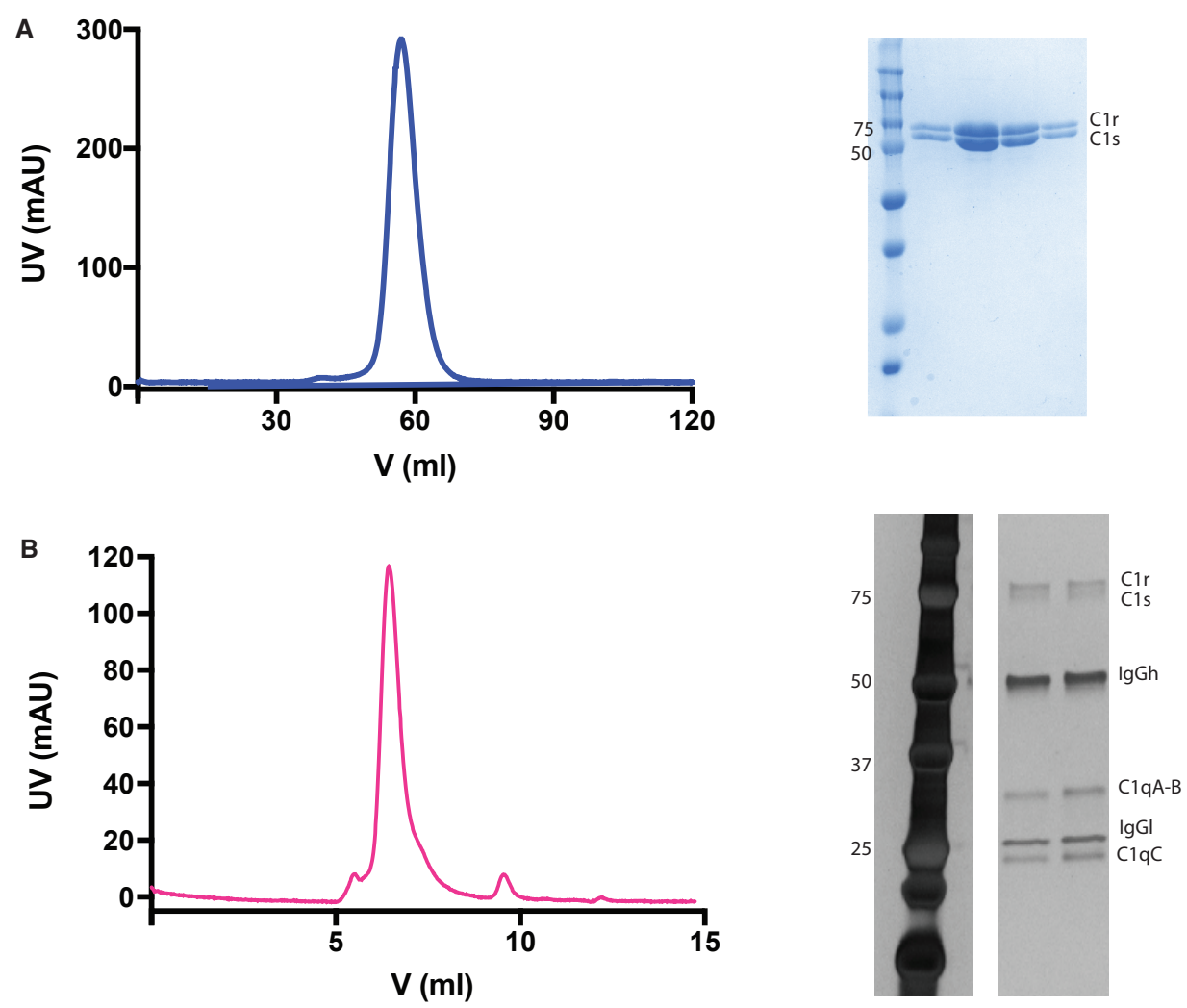

Supplementary Fig. S3. Sample preparation for single-particle cryo-EM.

(A) Gel-filtration profile of $\mathrm{Cl}_{2} \mathrm{~s}_{2}$ purified with a Superose 6 16/60 column. A Coomassiestained reducing SDS-PAGE gel is shown on the right. (B) Gel-filtration profile of C1-IgG16 purified with a BioSep 4000 column. Silver-stained reducing SDS-PAGE gel shows C1r, $\mathrm{C} 1 \mathrm{~s}$, IgG1 heavy chain (IgGh) and light chain(IgGl), C1q-A, B and C chains on the right. 
A

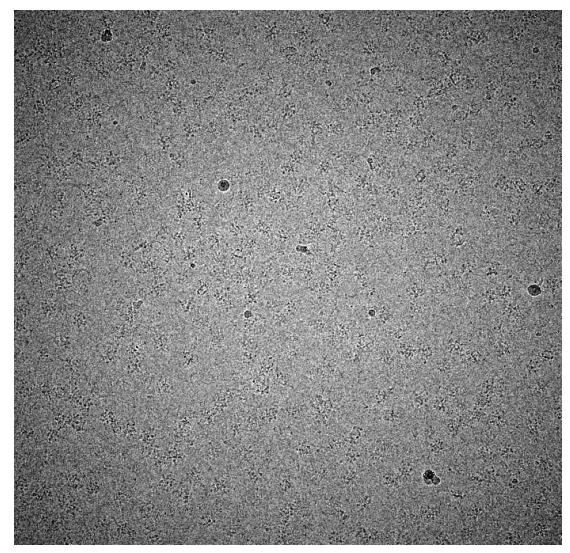

$$
\text { c }
$$

3357 micrographs

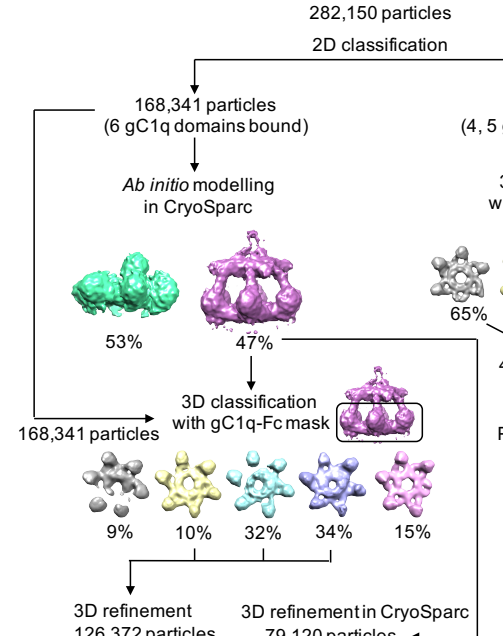

126,372 particles 79,120 particles$$
\text { 126,372 particles } 79,120 \text { particles }
$$

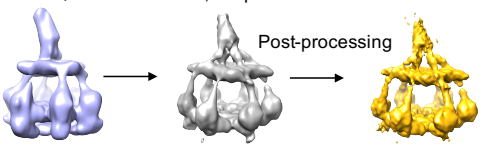

$$
\downarrow \quad \begin{gathered}
\text { 3D refinementin CryoSparc } \\
\text { with gC1q-Fc mask }
\end{gathered}
$$$$
\text { Ces } \rightarrow \text { Post-processing }
$$

E

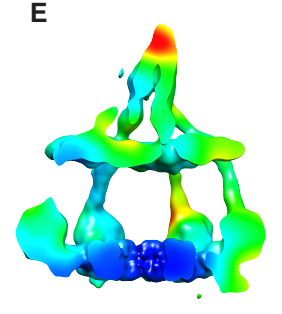

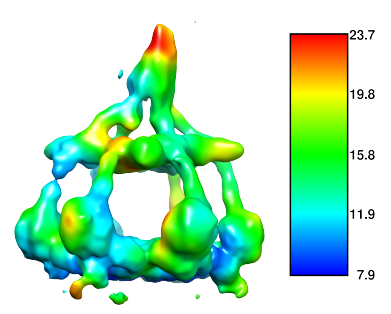

B
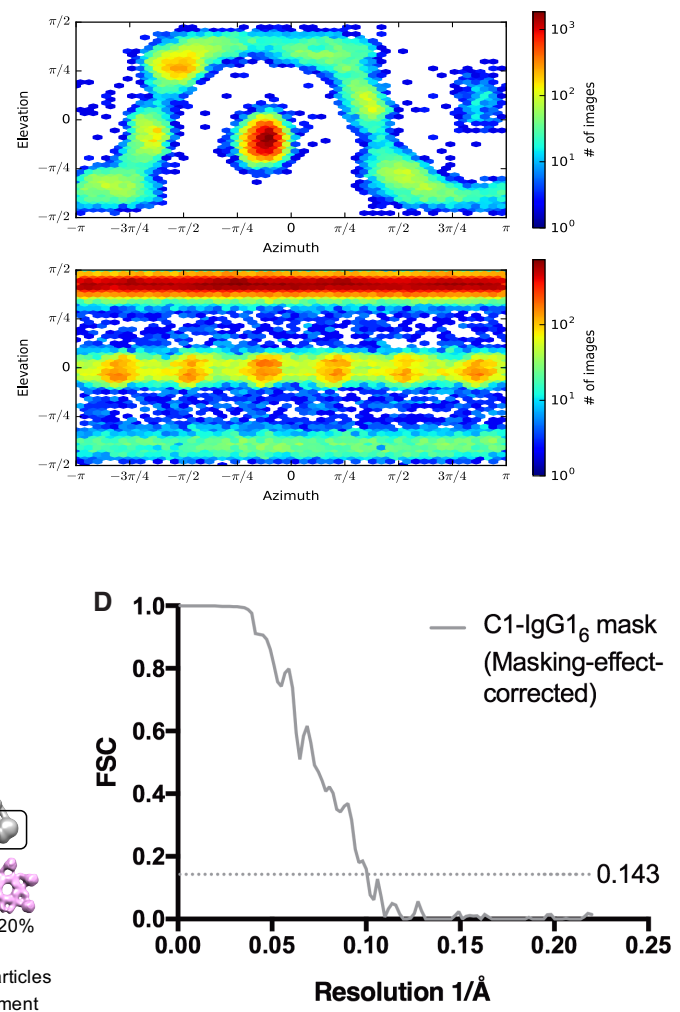
$3 \mathrm{D}$ refinement $3 \mathrm{D}$ refinement

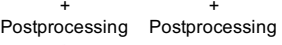

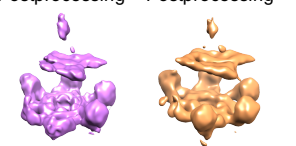

$4 \mathrm{gC} 1 \mathrm{q}$ bound $5 \mathrm{gC} 1 \mathrm{q}$ bound

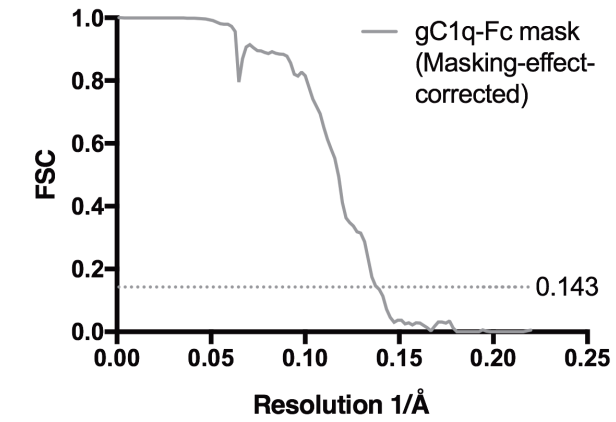

F

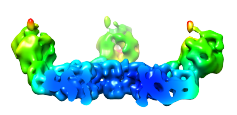

Supplementary Fig. S4. Cryo-EM single particle analysis of C1-IgG1 6 and single particle analysis workflow. 
(A) Representative micrograph of with a pixel size of $2.27 \AA$ and a defocus value of $-2.2 \mu \mathrm{m}$.

(B) Direction distribution plots of particles for non-symmetric C1-IgG1 6 map and C6symmetry imposed gC1q-Fc map. (C) Single particle reconstruction workflow. 2D classification, 3D classification, 3D refinement and postprocessing jobs were performed by Relion 2.0 except the jobs stated as "in Cryo Sparc". The mask applied around the gC1q-Fc region is shown as black box. The percentages of the particles for classification jobs are stated below the densities. (D) Fourier-shell correlation curve of C1-IgG1 6 map (top) and C6symmetry imposed gC1q-Fc map (bottom) (E) Single-particle reconstruction of C1-IgG1 6 colored by local resolution expressed in $\AA$ (F) C6-symmetry imposed single-particle reconstruction of $\mathrm{gC1q}$ units bound to Fc-platform colored by local resolution. 

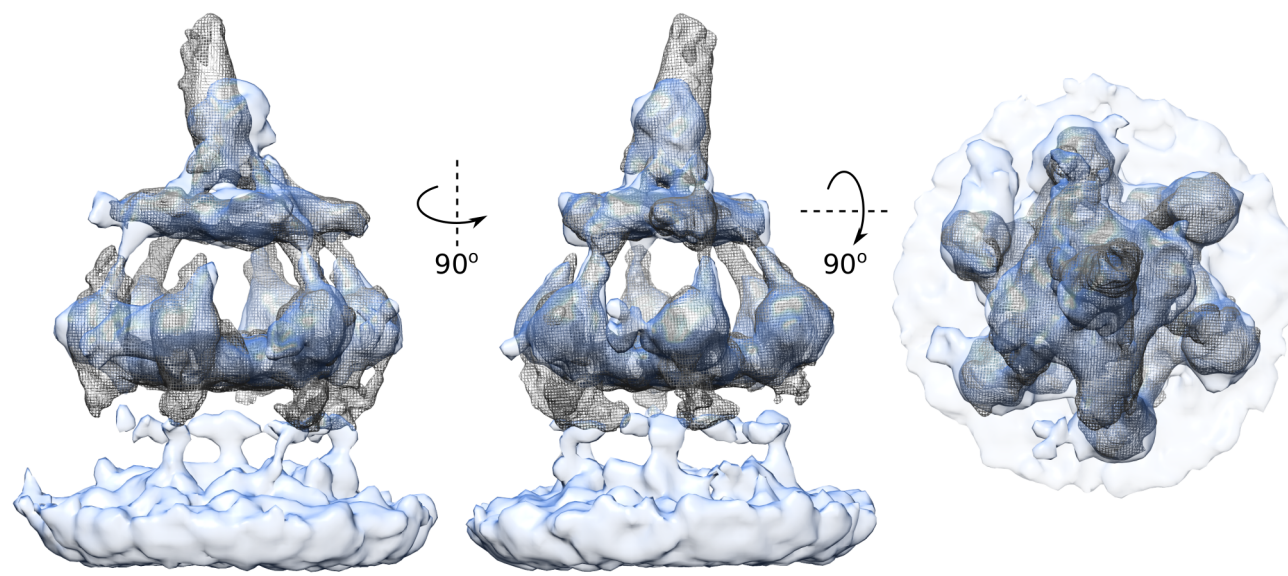

Supplementary Fig. S5. Comparing cryo-EM tomography and single-particle reconstructions.

Reconstructions derived from tomograms (blue surface; from Fig. 1C) and single-particle analysis (grey mesh; from Fig. 2C) aligned to show similarities in density from the side (left and middle) and top (right). 

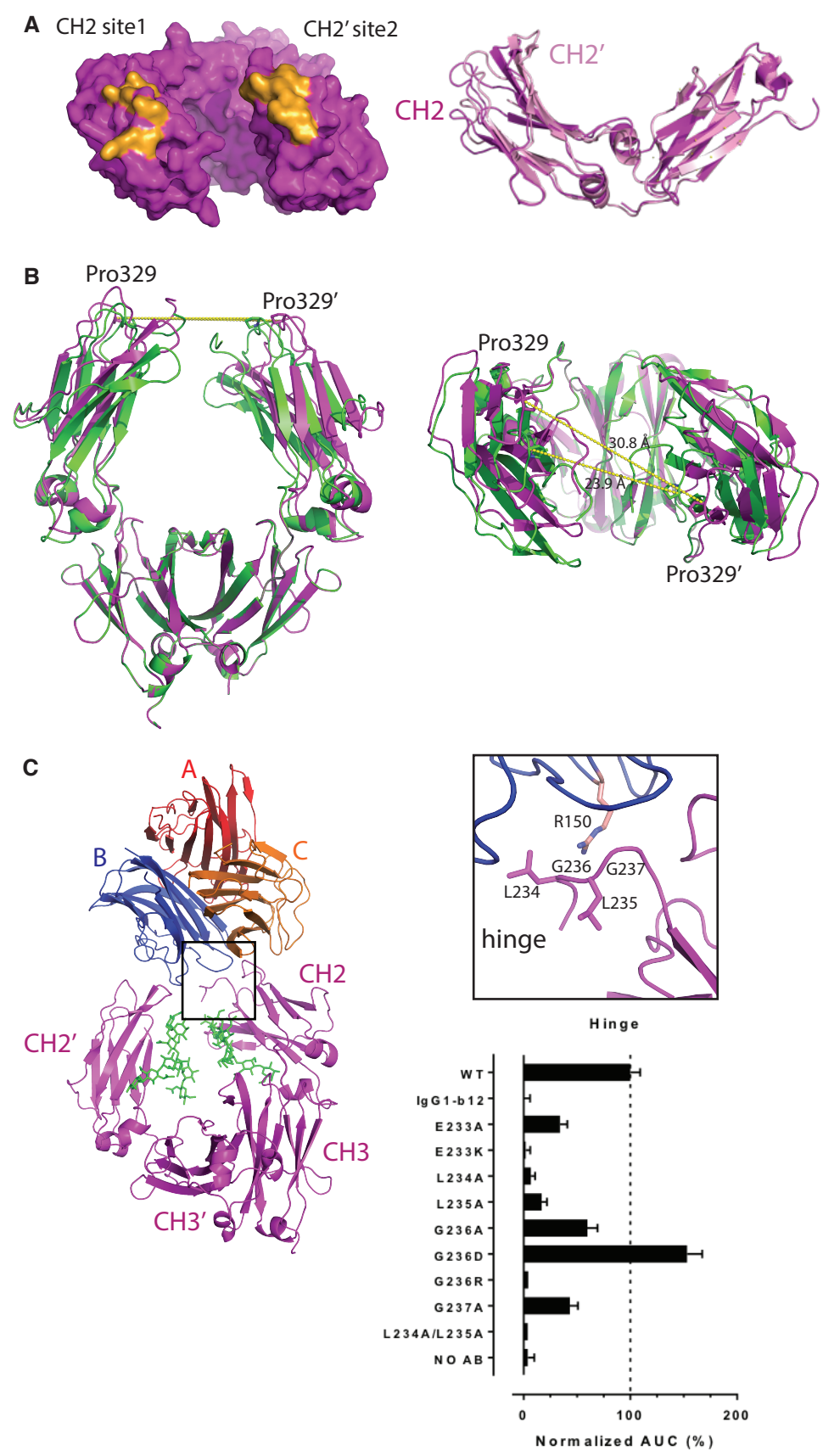

Supplementary Fig. S6. Identification of residues participating in gC1q-Fc interactions.

(A) Surface-area representation of Fc (magenta) with binding sites on $\mathrm{CH} 2$ and $\mathrm{CH} 2$ ' as footprint of $\mathrm{gClq}$ (orange) (left). Alignment of $\mathrm{CH} 2$ and $\mathrm{CH} 2$ ' in cartoon representation (right) (B) Fc (magenta) with a distance of 31 Å between Pro329-Pro329’ and Fc arrangement as observed in the IgG1- b12 crystal structure (1HZH) exhibiting Pro329Pro329' distance $23.8 \AA$ (green). (C) The Fab-Fc hinge region contributes to C1q binding. 
IgG Fc domain in magenta, glycans in green, C1q-A, B and C domains in red, blue, and orange respectively. Right panel: Complement dependent cytotoxicity assays of Raji cells opsonized with wild-type (WT) and mutated CD20 mAb IgG1-7D8 (n=3) exposed to C1qdeficient serum to which a titration of $1 \mathrm{ng} / \mathrm{mL}$ to $60 \mu \mathrm{g} / \mathrm{mL} \mathrm{Clq}$ was added. Cell lysis was assessed by flow cytometry using propidium iodide staining. Bars show the average area under the curve (AUC) for this dose response normalized against the AUC obtained with the unmutated WT IgG1-7D8 set to $100 \%$. 

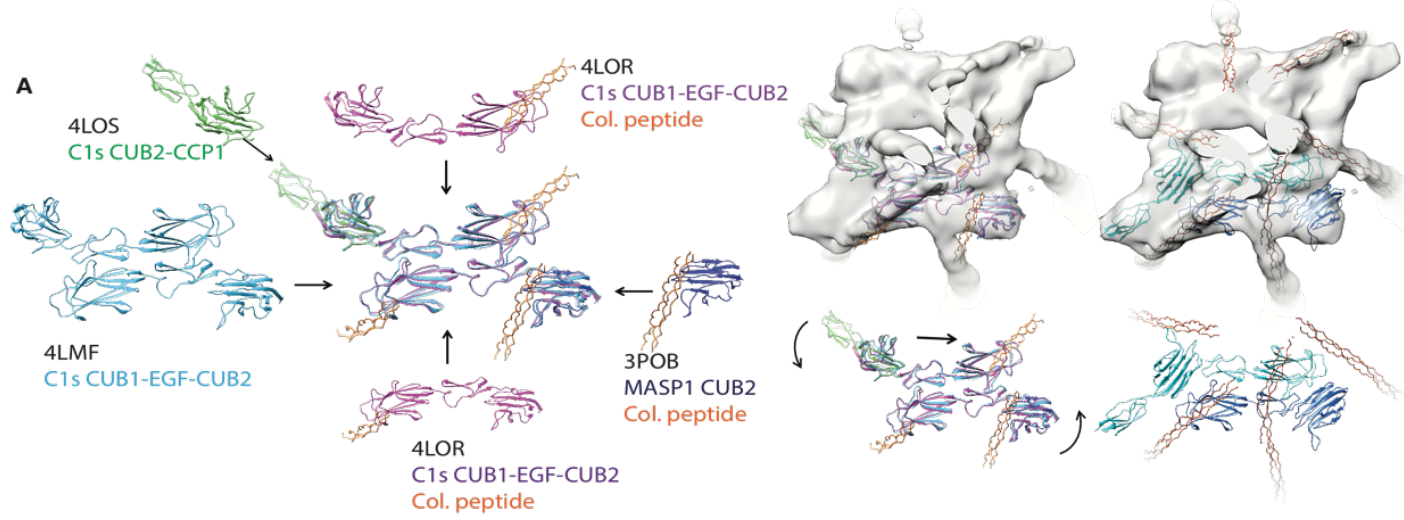

B
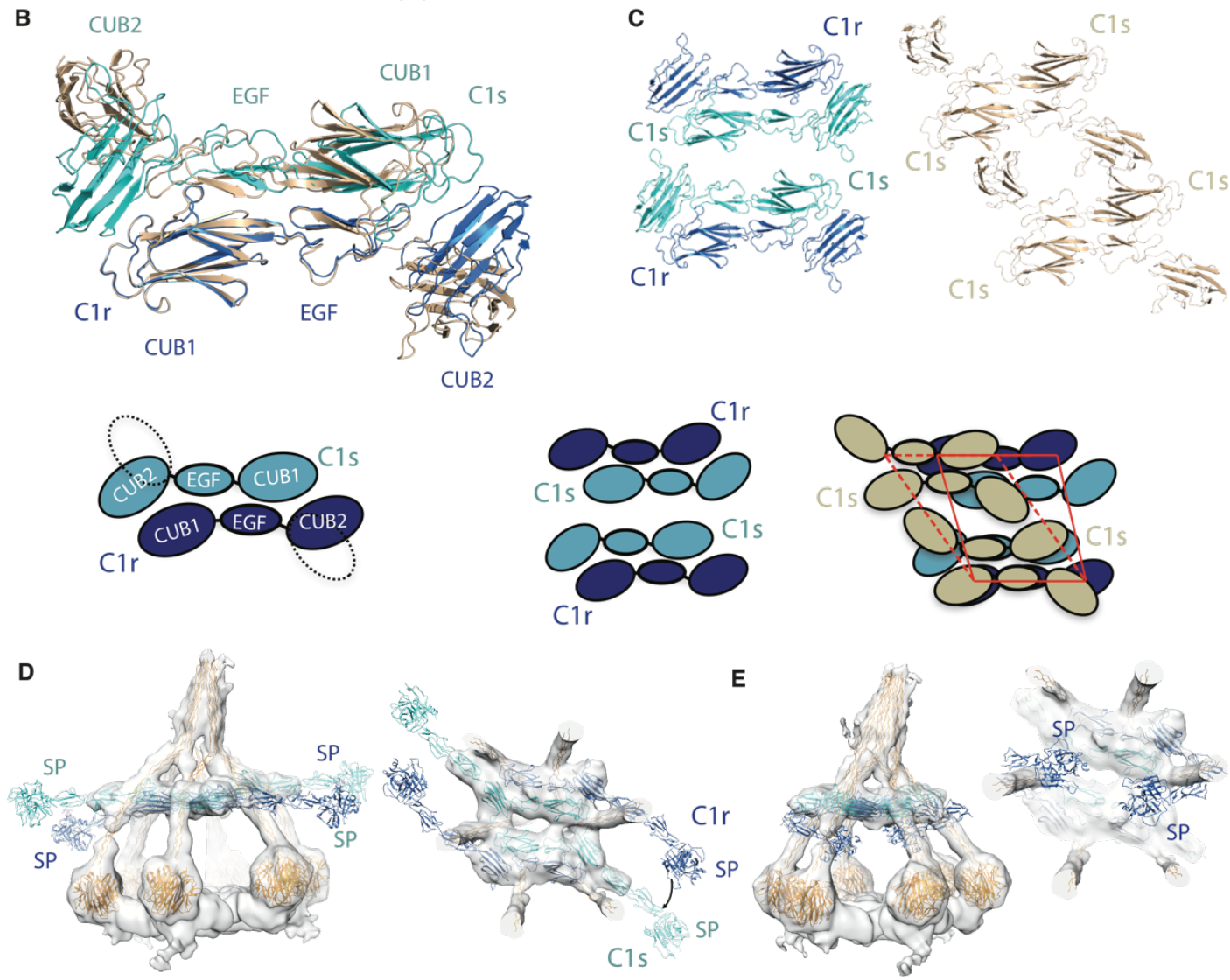

Supplementary Fig. S7. C1r and C1s arrangements.

(A) Crystal structures used for fitting of $\mathrm{C} 1 \mathrm{r}$ and $\mathrm{C} 1 \mathrm{~s}$ into the density. Initial positioning of 
each structure is shown on the left. The manual changes applied for fitting are shown by arrows. Final models (inside and outside the density) are shown on the right. (B) Superposition of the N-terminal CUB1-EGF-CUB2 domains of C1r (blue) and C1s (cyan) in C1-IgG1 6 onto the C1s CUB1-EGF-CUB2 arrangement (beige) observed in crystal structure 4LMF (25), indicating different orientations of CUB2 domains. (C) Comparison of C1s-C1s arrangements in $\mathrm{C} 1-\mathrm{IgG1}_{6}$ and the tetrameric packing of $\mathrm{C} 1 \mathrm{~s}$ observed in crystal structure 4LMF. Cartoon representations of the N-terminal CUB1-EGF-CUB2 arrangement for the $\mathrm{C} 1 \mathrm{r}-\mathrm{C} 1 \mathrm{~s}$ hetero-dimer in $\mathrm{C} 1-\mathrm{IgG} 1_{6}$ (left), with dashed line indicating the orientation of CUB2 domains in 4LMF, arrangement of the CUB1-EGF-CUB2 of C1r-C1s-C1s-C1r in C1-IgG1 6 (middle) and superposition of C1s CUB1-EGF-CUB2 stacking observed in 4LMF (right). Parallelograms in red solid and dashed lines indicate the effect of shifting the central $\mathrm{C} 1 \mathrm{~s}$ molecules by ca. $20 \AA$ sideways. (D) Hypothetical model obtained by extending CCP2-SP outwards in side (left) and bottom view (right). This arrangement, that is consistent with the observed density, supports the catalytic SP domain C1r to reach and cleave the scissile bond of the adjacent C1s. (E) Modeling C1r CCP2-SP oriented inwards for putative auto-activation in side view (left) and bottom view (right), indicating that the observed density of $\mathrm{C} 1-\mathrm{IgG} 1_{6}$ is not consistent with an intra-complex auto-activation of $\mathrm{C} 1 \mathrm{r}$ by the opposing $\mathrm{C} 1 \mathrm{r}$. Generation of a C1r-C1r enzyme-substrate arrangement within a single $\mathrm{C} 1$ would require domain rearrangements incongruent with the observed density. Moreover, no density is observed in between the N-terminal protease platform and Fc platform in our data (both from tomography and single-particle analysis). 
Supplementary Table S1

\begin{tabular}{|c|c|c|c|c|c|c|c|}
\hline \multicolumn{8}{|c|}{ Experimental series 1 } \\
\hline Antibody & Location & $\begin{array}{c}\text { Mean } \\
\text { AUC }(\%) \\
(1)\end{array}$ & $\begin{array}{l}\text { SD }(\%) \\
\quad(2)\end{array}$ & $\begin{array}{l}\mathbf{N} \\
(3)\end{array}$ & \multicolumn{3}{|c|}{$\begin{array}{l}\text { One way ANOVA, Dunnett's multiple } \\
\text { comparisons test }\end{array}$} \\
\hline WT IgG1-7D8 & Control & 100.0 & 9.2 & 3 & \multirow{2}{*}{$\begin{array}{c}\text { Mean } \\
\text { difference }\end{array}$} & \multirow{2}{*}{$\begin{array}{l}\text { 95\% CI of } \\
\text { difference }\end{array}$} & \multirow{2}{*}{$\begin{array}{c}\text { Adjusted } p \\
\text { value (4) }\end{array}$} \\
\hline IgG1 b12 & Control & 0.0 & 5.9 & 3 & & & \\
\hline E233A & Hinge & 34.0 & 6.8 & 3 & 66 & 40.7 to 91.3 & $<0.0001$ \\
\hline E233K & Hinge & 1.8 & 4.3 & 3 & 98.2 & 72.9 to 123 & $<0.0001$ \\
\hline L234A & Hinge & 6.8 & 4.0 & 3 & 93.2 & 67.9 to 119 & $<0.0001$ \\
\hline $\mathrm{L} 235 \mathrm{~A}$ & Hinge & 16.9 & 5.0 & 3 & 83.1 & 57.8 to 108 & $<0.0001$ \\
\hline G236A & Hinge & 59.7 & 9.5 & 3 & 40.3 & 15 to 65.6 & $<0.0001$ \\
\hline G236D & Hinge & 153.1 & 14.2 & 3 & -53.1 & -78.4 to -27.8 & $<0.0001$ \\
\hline G236R & Hinge & 4.2 & 3.4 & 3 & 95.8 & 70.5 to 121 & $<0.0001$ \\
\hline G237A & Hinge & 43.4 & 7.4 & 3 & 56.6 & 31.3 to 81.9 & $<0.0001$ \\
\hline E269A & Site 2 & 32.0 & 7.2 & 3 & 68 & 42.7 to 93.3 & $<0.0001$ \\
\hline E269K & Site 2 & 10.7 & 8.5 & 3 & 89.3 & 64 to 115 & $<0.0001$ \\
\hline E294A & Site 2 & 82.0 & 7.3 & 3 & 18 & -7.25 to 43.3 & 0.3575 \\
\hline E294K & Site 2 & 24.1 & 7.9 & 3 & 75.9 & 50.6 to 101 & $<0.0001$ \\
\hline S298A & Site 2 & 110.6 & 9.7 & 3 & -10.6 & -35.9 to 14.7 & 0.9629 \\
\hline S298K & Site 2 & 14.1 & 7.0 & 3 & 85.9 & 60.6 to 111 & $<0.0001$ \\
\hline Y300A & Site 2 & 145.6 & 11.8 & 3 & -45.6 & -70.9 to -20.4 & $<0.0001$ \\
\hline Y300D & Site 2 & 207.2 & 13.0 & 3 & -107 & -133 to -81.9 & $<0.0001$ \\
\hline Y300R & Site 2 & 26.8 & 11.3 & 3 & 73.2 & 48 to 98.5 & $<0.0001$ \\
\hline K322A & Site 1 & 15.4 & 6.9 & 3 & 84.6 & 59.3 to 110 & $<0.0001$ \\
\hline A327G & Site 1 & 145.6 & 10.6 & 3 & -45.6 & -70.9 to -20.4 & $<0.0001$ \\
\hline A327E & Site 1 & 100.0 & 9.5 & 3 & 0 & -25.3 to 25.3 & 0.9999 \\
\hline A327K & Site 1 & 13.3 & 6.8 & 3 & 86.7 & 61.4 to 112 & $<0.0001$ \\
\hline No $\mathrm{Ab}$ & Control & 3.8 & 6.1 & 3 & 96.2 & 70.9 to 121 & $<0.0001$ \\
\hline
\end{tabular}

\begin{tabular}{|c|c|c|c|c|c|c|c|}
\hline \multicolumn{8}{|c|}{ Experimental series 2} \\
\hline Antibody & Location & $\begin{array}{c}\text { Mean } \\
\text { AUC (\%) } \\
(1)\end{array}$ & $\begin{array}{c}\text { SD } \\
(\%)(2)\end{array}$ & $\begin{array}{l}\mathbf{N} \\
(\mathbf{3})\end{array}$ & \multicolumn{3}{|c|}{$\begin{array}{l}\text { One way ANOVA, Dunnett's multiple } \\
\text { comparisons test }\end{array}$} \\
\hline WT IgG1-7D8 & Control & 100.0 & 10.6 & 6 & \multirow{2}{*}{$\begin{array}{c}\text { Mean } \\
\text { difference }\end{array}$} & \multirow{2}{*}{$\begin{array}{l}95 \% \text { CI of } \\
\text { difference }\end{array}$} & \multirow{2}{*}{$\begin{array}{l}\text { Adjusted p } \\
\text { value (4) }\end{array}$} \\
\hline IgG1 b12 & Control & 0.0 & 4.2 & 6 & & & \\
\hline H268Q & Site 2 & 75.4 & 7.3 & 3 & 24.6 & 6.39 to 42.7 & 0.0031 \\
\hline $\mathrm{D} 270 \mathrm{~A}$ & Site 2 & -0.7 & 3.0 & 3 & 101 & 82.5 to 119 & $<0.0001$ \\
\hline N297Q & Site 2 & -0.4 & 2.6 & 3 & 100 & 82.2 to 119 & $<0.0001$ \\
\hline K322A & Site 1 & -0.4 & 2.7 & 3 & 100 & 82.2 to 119 & $<0.0001$ \\
\hline P329A & Site 1 & -2.0 & 2.9 & 3 & 102 & 83.9 to 120 & $<0.0001$ \\
\hline P331A & Site 1 & 12.9 & 6.7 & 3 & 87.1 & 68.9 to 105 & $<0.0001$ \\
\hline L234A/L235A & Hinge & 3.8 & 4.8 & 3 & 96.2 & 78 to 114 & $<0.0001$ \\
\hline K326A/E333A & Site 1 & 223.7 & 11.6 & 3 & -124 & -142 to -105 & $<0.0001$ \\
\hline No Ab & Control & -0.7 & 3.8 & 3 & 101 & 82.6 to 119 & $<0.0001$ \\
\hline
\end{tabular}


Supplementary Table 1: Area under the curve (AUC) values for CDC of Raji cells normalized relative to the WT IgG1-7D8, which was set at 100\% lysis. CDC data were collected in in two independent series of three experiments, and were normalized per series (top panel, bottom panel). Mean area under the curve (AUC) (1), standard deviation (SD) (2), number of replicates (N) (3), and multiplicity adjusted p-value of statistical analysis (4) are shown. One-way ANOVA was followed by Dunnett's Multiple Comparison Posthoc Test using GraphPad Prism 7.02. A p-value $<0.05$ indicates that the CDC induced by a mutant was different from that determined for wild-type IgG1-7D8.

\section{References}

33. J. L. Teeling et al., Characterization of new human CD20 monoclonal antibodies with potent cytolytic activity against non-Hodgkin lymphomas. Blood. 104, 1793-1800 (2004).

34. K. D. White, M. B. Frank, S. Foundling, F. J. Waxman, Effect of immunoglobulin variable region structure on $\mathrm{C} 3 \mathrm{~b}$ and $\mathrm{C} 4 \mathrm{~b}$ deposition. Mol. Immunol. 33, 759-768 (1996).

35. X. Fan et al., Near-Atomic Resolution Structure Determination in Over-Focus with Volta Phase Plate by Cs-corrected Cryo-EM. bioRxiv. 25, 1623-1630.e3 (2017).

36. X. Li et al., Electron counting and beam-induced motion correction enable nearatomic-resolution single-particle cryo-EM. Nat. Methods. 10, 584-90 (2013).

37. D. N. Mastronarde, S. R. Held, Automated tilt series alignment and tomographic reconstruction in IMOD. J. Struct. Biol. 197, 102-113 (2017).

38. D. Castaño-Díez, M. Kudryashev, H. Stahlberg, Dynamo Catalogue: Geometrical tools and data management for particle picking in subtomogram averaging of cryo-electron tomograms. J. Struct. Biol. 197, 135-144 (2017).

39. G. Tang et al., EMAN2: An extensible image processing suite for electron microscopy. J. Struct. Biol. 157, 38-46 (2007). 
40. A. Rohou, N. Grigorieff, CTFFIND4: Fast and accurate defocus estimation from electron micrographs. J. Struct. Biol. 192, 216-221 (2015).

41. S. H. W. Scheres, Semi-automated selection of cryo-EM particles in RELION-1.3. J. Struct. Biol. 189, 114-22 (2015).

42. S. H. W. Scheres, RELION: Implementation of a Bayesian approach to cryo-EM structure determination. J. Struct. Biol. 180, 519-530 (2012).

43. A. Punjani, J. L. Rubinstein, D. J. Fleet, M. A. Brubaker, cryoSPARC: algorithms for rapid unsupervised cryo-EM structure determination. Nat. Methods. 14, 290-296 (2017).

44. E. F. Pettersen et al., UCSF Chimera-A Visualization System for Exploratory Research and Analysis. J Comput Chem. 25, 1605-1612 (2004).

45. J. Kopp, T. Schwede, The SWISS-MODEL Repository of annotated three-dimensional protein structure homology models. Nucleic Acids Res. 32, D230-4 (2004).

46. I. Farabella et al., TEMPy: A Python library for assessment of three-dimensional electron microscopy density fits. J. Appl. Crystallogr. 48, 1314-1323 (2015).

47. S. J. Perkins, A. S. Nealis, B. J. Sutton, A. Feinstein, Solution structure of human and mouse immunoglobulin $\mathrm{M}$ by synchrotron X-ray scattering and molecular graphics modelling. A possible mechanism for complement activation. J. Mol. Biol. 221, 1345$1366(1991)$. 